\title{
Simulation of Progressive Failure in Multidirectional Composite Laminated Panels *
}

\author{
Shiladitya Basu ${ }^{\dagger}$ and Anthony M. Waas ${ }^{\ddagger}$ \\ University of Michigan, Ann Arbor, Michigan, 48109 \\ Damodar R. Ambur $\S^{\S}$ \\ NASA Glenn Research Center, Cleveland, $\mathrm{OH} 44135$
}

\begin{abstract}
A mechanism-based progressive failure analyses (PFA) approach is developed for fiber reinforced composite laminates. Each ply of the laminate is modeled as a nonlinear elastic degrading lamina in a state of plane stress according to Schapery theory. In this theory, each lamina degrades as characterized through laboratory scale experiments. In the fiber direction, elastic behavior prevails, however, in the present work, the phenomenon of fiber microbuckling, which is responsible for the sudden degradation of the axial lamina properties under compression, is explicitly accounted for by allowing the fiber rotation at a material point to be a variable in the problem. The latter is motivated by experimental and numerical simulations that show that local fiber rotations in conjunction with a continuously degrading matrix are responsible for the onset of fiber microbuckling leading to kink banding. These features are built into a user defined material subroutine that is implemented through the commercial finite element (FE) software ABAQUS. The present model, thus, disbands the notion of a fixed compressive strength of a lamina and instead uses the mechanics of the failure process to provide the in-situ compression strength of a material point in a lamina, the latter being dictated strongly by the current local stress state, the current state of the lamina transverse material properties and the local fiber rotation. The inputs to the present work are laboratory scale, coupon level test data that provide information on the lamina transverse property degradation (i.e. appropriate, measured, strain-stress relations of the lamina transverse properties), the elastic lamina orthotropic properties, the ultimate tensile strength of the lamina in the fiber direction and the geometry of the structural panel. The validity of the approach advocated is demonstrated through numerical simulations of the response of two composite structural panels that are loaded to complete failure. A flat, 24-ply unstiffened panel with a cutout subjected to in-plane shear loading, and a double notched 70 ply unstiffened stitched panel subjected to axial compression are selected for study. The predictions of the simulations are compared against experimental data. Good agreement between the present PFA and the experimental data are reported.
\end{abstract}

*This paper is dedicated to the memory of Dr. James H. Starnes, Jr., for sustained and life long contributions to composite structural mechanics and especially for his contributions to the subject of structural stability.

${ }^{\dagger}$ Doctoral candidate, University of Michigan, member, AIAA.

$\ddagger$ Professor, author to whom all correspondence should be addressed. (dcw@umich.edu, Tel:734-764-8227, Fax:734-763-0578), Associate Fellow, AIAA.

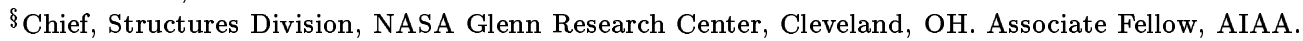

Copyright (C) 2005 by Anthony M. Waas. Published by the American Institute of Aeronautics and Astronautics, Inc. with permission. 


\section{Introduction}

Development of computational methodologies for the prediction of damage accumulation and growth in continuous fiber composite laminates is presently an active area of research. A large body of literature devoted to progressive failure analysis (PFA) of composite laminated structures is now present. Many of the PFA schemes introduced and available today have relied on the phenomenological approach of defining strength criteria for a single lamina when subjected to different single component stress states. These methods define the onset of failure through specific indices that are expressed as functions of the current stress state. When any of these indices exceeds a predefined critical value, the material at that point is said to have failed. ${ }^{1,2}$ When a material point has failed, for subsequent loading, it is assumed to have a reduced stiffness that is predetermined in an empirical manner. Depending on the type of failure (for instance, fiber breaking and/or matrix cracking due to tension along the fibers, fiber kink-banding due to compression along the fibers, fiber/matrix debonding due to in-plane shear), different elastic moduli are set to zero. In addition, linear elastic material behavior is assumed throughout the analysis. In a laminated composite plate, the stiffness at a material point is determined by the current local stress state and the local 'state' of the material. The local stress state, in general, is multi-axial and a material point at the current state may have accumulated damage, dictated by the loading history. Thus, 'strength' at a material point is influenced to a great extent by the current stress and strain state and is predicated on the mechanism of failure. Schemes that abruptly change material properties and rely on a linear elastic analysis may be unable to realistically account for features associated with the mechanisms of failure. Furthermore, these approaches lack an appropriate definition of the material state beyond first failure. For progressive failure modeling, a framework that accounts for the continued degradation of the material is needed. Schapery ${ }^{3}$ introduced a thermodynamically based theory (referred to as ST) that uses internal state variables (ISVs) to analyze damage evolution in composite laminates. These ISVs are related to mechanical aspects of damage mechanisms. The ISVs are related to the energy required for the evolution of the damaged states. Different damage mechanisms can have distinct ISVs to track the damage evolution. For instance, matrix microcracking can be expressed via one ISV, while transverse cracking can be represented by another ISV. ${ }^{4}$ The evolution of these ISVs with global loading is determined at each material point and as functions of load history through the satisfaction of a thermodynamic criterion (an evolution equation), throughout the loading history. Various experimental ${ }^{5}$ and analytical ${ }^{6}$ studies show that the maximum load sustained by a lamina in the axial direction $\left(\sigma_{11}^{c r}\right)$, in compression, depends greatly on the presence of the other in-plane stress components such as $\sigma_{22}$ and $\tau_{12}$. Experimental, analytical and micromechanical studies show that the main physical event occurring during kinking is the rotation of fibers within a degrading matrix. The rotation of fibers gives rise to high localized shear strains which drives the shear degradation of the local matrix. The shear degradation in turn drives the rotation of the fibers creating a positive feedback loop. The simultaneous presence of both these phenomena results in a limit load situation for a lamina under axial compression. This limiting load, which is dictated by the local stress state and the state of the transverse lamina properties, can be relieved (increased) by the presence of other stress components or, in certain cases, can be elevated (reduced). The present approach captures these two phenomena in a numerical setting and is able to reproduce the broad micromechanical observations associated with compressive failure at a macrolevel (lamina level, instead of at the individual fiber/matrix level). Two structural panel configurations, for which a set of laboratory data is available, are studied using the present PFA approach. A thick (70-ply), stitched double notched carbon fiber reinforced laminated panel subjected to axial compression loading and a relatively thin (24-ply) flat unstiffened laminated shear panel (FSP) with a cutout, loaded deep into the postbuckling regime are analyzed. Double notched panels (DNPs), with notch tip radii equal to the laminate thickness are cut from a master laminate (Figure 1). The notches act as stress concentrators and create a high stress zone, making it easier to observe damage initiation and progression. The use of a stitched laminate eliminates delamination among adjacent plies of a multidirectional laminate making it possible to isolate matrix dominated failure events in an uncomplicated manner. The unnotched sides of the DNPs are subjected to remote uniaxial displacement control compression loading. The notched sides are placed in 
anti-buckling guides to prevent premature global buckling. Figure 2 shows the loading scheme and boundary conditions. Global load-deflection data and damage initiation and progression data are obtained from the laboratory experiments, through strain gages, load cells, LVDT's and post-experiment microscopic studies. The experiments show that fiber kinking in the zero plies (plies parallel to the loading direction) is the dominant mode of failure and is seen to occur at the vicinity of the notch tip within the zero plies. The kinking zone subsequently grows towards the center of the panel resulting in global catastrophic failure. For the flat unstiffened, 24-ply panel, experimental results and related PFA modeling has been reported earlier ${ }^{7-9}$. These panels are loaded in shear using a picture frame test set-up. Back to back strain gage data and load and load point displacement data from the panel tests are used to validate the present PFA approach.

The main objective of the present paper is to introduce a new PFA approach for composite laminates that is based on capturing features associated with failure mechanisms and embedding these in a numerical scheme that can model the laminate as a collection of degrading lamina. The selected examples account for both material degradation in a nonlinear manner (DNP), and the interaction of these nonlinearities with geometrical nonlinearities (FSP) which dominate the response of thin-gage structures that are loaded in compression.

\section{Progressive Failure Analysis using Schapery Theory (ST)}

\section{A. Elements of the PFA approach}

\section{Non-linear Constitutive Formulation}

Schapery ${ }^{10}$ developed nonlinear elastic constitutive relations for an orthotropic lamina using a work potential approach which accounted for the effect of microdamage. The lamina stress-strain relations are,

$$
\begin{aligned}
\sigma_{11} & =Q_{11} \epsilon_{11}+Q_{12} \epsilon_{22} \\
\sigma_{22} & =Q_{12} \epsilon_{11}+Q_{22} \epsilon_{22} \\
\tau_{12} & =Q_{66} \gamma_{12}
\end{aligned}
$$

where,

$$
\begin{array}{rll}
Q_{11}=\frac{E_{11}}{1-\nu_{12} \nu_{21}} & ; \quad Q_{22}=\frac{E_{22}}{1-\nu_{12} \nu_{21}} \\
Q_{12}=\nu_{12} Q_{22} & ; \quad Q_{66}=G_{12} \\
\nu_{21} & =\frac{\nu_{12} E_{22}}{E_{11}}
\end{array}
$$

In the existing literature on damage mechanics as applied to continuous fiber laminated composite materials, the effect of damage is incorporated through the change in transverse Young's modulus $E_{22}$ and in plane shear modulus $G_{12}$. For instance, Sun and $\mathrm{Chen}^{11}$ proposed a one parameter plastic potential in conjunction with orthotropic incremental plasticity theory to study the evolution of $E_{22}$ and $G_{12}$ in tension. Schapery and Sicking, ${ }^{4}$ used ST to study the evolution of $E_{22}$ and $G_{12}$. These previous studies were not concerned with the state of the lamina beyond first failure in the fiber direction. Yet, it is recognized ${ }^{12}$ that such damage is dominant for compression loaded composite structures. Lamina level coupon tests in tension have shown that fiber direction modulus, $E_{11}$ and Poissons ratio, $\nu_{12}$ can be assumed to be independent of microdamage that influence $E_{22}$ and $G_{12} \cdot{ }^{13}$ This situation is also true for compression until the onset of kinking (the axial compression load reaches a maximum limit load at the point in which a kink band starts to form, for example, as shown in Lee and Waas ${ }^{14}$ ). During kink band formation and propagation, it is likely that microdamage mechanisms do influence $E_{11}, \nu_{12}, E_{22}$ and $G_{12}$. Subsequent to kink banding, other failure mechanisms such as delamination can occur. These mechanisms are not accounted for here, but have received attention recently in the context of $\mathrm{PFA} .^{9}$ 


\section{Elements of Schapery Theory}

In Schapery and Sicking, ${ }^{4}$ ISVs are used to incorporate inelastic behavior in the material response. Earlier, Schapery ${ }^{3}$ introduced a more general thermodynamic framework to study materials that undergo damage. In these developments, the total work done, $W_{T}$, in a mechanical process is composed of the inelastic work, $W_{s}$ and the work of deformation $W$.

$$
W_{T}=W+W_{s}
$$

The irrecoverable portion of total energy $\left(W_{s}\right)$ can be determined from the material stress-strain response as shown in Figure 3. $I S V$ s are described through $S_{i}$ 's. Each $S_{i}$ is associated with a particular damage mechanism. To satisfy the path independence of total work, these $I S V$ s have to satisfy the following relation,

$$
f_{i}=\frac{\partial W_{s}}{\partial S_{i}}
$$

The left hand side of Eq. (4) is referred to as the thermodynamic force related to the $i^{\text {th }} I S V$. If the $i^{\text {th }}$ driving force, which is the available thermodynamic force, given by Eq. (5), exceeds $\frac{\partial W_{s}}{\partial S_{i}}$, then the material undergoes a structural change that is associated with $S_{i}$.

$$
f_{i} \equiv-\frac{\partial W}{\partial S_{i}}
$$

Stated another way,

$$
\dot{S}_{i}>0
$$

However, as pointed out by Schapery,${ }^{3}$ it is to be noted that if the available thermodynamic force, $\left(-\frac{\partial W}{\partial S_{i}}\right)$ is less than the required thermodynamic force $\left(\frac{\partial W_{s}}{\partial S_{i}}\right)$ then,

$$
\dot{S}_{i}=0
$$

Furthermore, $S_{i}$ need not change continuously with the loading.

Schapery considered two $I S V$ s. They were the energies associated with matrix microcracks $(S)$ and of the transverse intra-ply cracks $\left(S_{c}\right)$, respectively. Inelastic work is described as,

$$
W_{s}=S+S_{c}
$$

These ISVs affect the moduli $E_{22}$ and $G_{12}$ through Eq. (9)

$$
\begin{aligned}
E_{22} & =E_{22_{0}} e_{s}(S) e_{c}\left(S_{c}\right) \\
G_{12} & =G_{12_{0}} g_{s}(S) g_{c}\left(S_{c}\right)
\end{aligned}
$$

Here, $E_{22_{0}}$ and $G_{12_{0}}$ are transverse and shear moduli of the virgin material, i.e., at zero strain and zero damage; $e_{s}(S)$ and $g_{s}(S)$ are functions relating these two moduli to microdamage ISV, $S$ and $e_{c}\left(S_{c}\right)$ and $g_{c}\left(S_{c}\right)$ are functions relating $E_{22}$ and $G_{12}$ to the transverse cracking ISV, $S_{c}$. The functions $e_{s}, e_{c}, g_{s}$ and $g_{c}$ are expressed as polynomial relations in the respective ISVs. ${ }^{4}$

The strain energy density (or work of deformation) can be written as

$$
W=\frac{1}{2}\left(Q_{11} \epsilon_{11}^{2}+Q_{22} \epsilon_{22}^{2}\right)+\nu_{12} Q_{22} \epsilon_{11} \epsilon_{22}+\frac{G_{12} \gamma_{12}^{2}}{2}
$$

To incorporate geometric nonlinearities, Green's strains and the second Piola Kirchoff stresses need to be used in the expression for $W$. For small strains, Eq. (10), would contain only the first order terms 
in the strain-displacement relations. Schapery and Sicking ${ }^{4}$ have shown that material non-linearities as incorporated in Eq. (10) are still significant for fiber reinforced composites even when inclusion of geometric nonlinearities are not called for. ${ }^{15}$

Using Eqs (1)-(5), the evolution equations for $S$ and $S_{c}$ are as follows,

$$
\begin{aligned}
& \frac{1}{2} \epsilon_{11}^{2} \frac{\partial Q_{11}}{\partial S}+\left(\frac{1}{2} \epsilon_{22}^{2}+\nu_{12} \epsilon_{11} \epsilon_{22}\right) \frac{\partial Q_{22}}{\partial S}+\frac{\gamma_{12}^{2}}{2} \frac{\partial G_{12}}{\partial S}=-1 \\
& \frac{1}{2} \epsilon_{11}^{2} \frac{\partial Q_{11}}{\partial S_{c}}+\left(\frac{1}{2} \epsilon_{22}^{2}+\nu_{12} \epsilon_{11} \epsilon_{22}\right) \frac{\partial Q_{22}}{\partial S_{c}}+\frac{\gamma_{12}^{2}}{2} \frac{\partial G_{12}}{\partial S_{c}}=-1
\end{aligned}
$$

In the present work, it is assumed that the fiber direction stiffnesses are unaffected by $S$ and $S_{c}$. Thus, the first terms in these equations can be neglected. Also, $\nu_{12} \nu_{21} \ll 1$. Thus, the above equations reduce to,

$$
\begin{aligned}
& \left(\frac{1}{2} \epsilon_{22}^{2}+\nu_{12} \epsilon_{11} \epsilon_{22}\right) \frac{\partial E_{22}}{\partial S}+\frac{\gamma_{12}^{2}}{2} \frac{\partial G_{12}}{\partial S}=-1 \\
& \left(\frac{1}{2} \epsilon_{22}^{2}+\nu_{12} \epsilon_{11} \epsilon_{22}\right) \frac{\partial E_{22}}{\partial S_{c}}+\frac{\gamma_{12}^{2}}{2} \frac{\partial G_{12}}{\partial S_{c}}=-1
\end{aligned}
$$

For an inelastic process, the entropy production rate is non-negative. Hence,

$$
\dot{S}+\dot{S}_{c} \geq 0
$$

The overdots represent temporal derivatives. Physically, $\dot{S}$ and $\dot{S}_{c}$ are both non-negative because healing (or reversible damage) is not allowed for in the damage mechanisms considered. Thus the ISVs individually satisfy Eq. (13), as well.

From experiments, ${ }^{4}$ it has been observed that for small strains, $\mathrm{S}$ behaves as $\epsilon^{3}$. This is based on the fact that moduli are constant for small strains. Thus to express the moduli, $E_{22}$ and $G_{12}$ in terms of a polynomial of $S$, a reduced variable $S_{r}$ can be used,

$$
S_{r} \equiv S^{1 / 3}
$$

The evolution equation for $S_{r}$ now becomes,

$$
\left(\frac{1}{2} \epsilon_{22}^{2}+\nu_{12} \epsilon_{11} \epsilon_{22}\right) \frac{\partial E_{22}}{\partial S_{r}}+\frac{\gamma_{12}^{2}}{2} \frac{\partial G_{12}}{\partial S_{r}}=-3 S_{r}^{2}
$$

The effect of transverse inter-ply cracking is not as easily measurable as the effect of microdamage due to matrix microcracking. An estimate can be obtained using Eq. (15) to approximate $S_{r}$, and then using the following relation,

$$
S_{c}=W_{T}-W-S
$$

In the present study, it is assumed that matrix microcracking is the only responsible mechanism for transverse property degradation. Consequently, Eq. (15) is the only relevant evolution equation. A detailed discussion of obtaining $S_{c}$ is provided by Schapery and Sicking. ${ }^{4}$ It should be noted that it is possible to include other softening damage mechanisms such as local fiber-matrix debonding and shear banding through $S$ as has been discussed by Schapery. ${ }^{3}$

\section{Fiber rotation under axial compression}

Results from previous experimental, analytical and numerical studies have shown that the main feature of laminate failure under predominantly compression loading is fiber kinking. The presence of initial fiber misalignment in a lamina that experiences compression along the fibers gives rise to local shear strain in the matrix adjacent to the misaligned fibers. These shear strains accelerate degradation of the local matrix 
shear stiffness. Imperfect fiber systems are prone to rotate under axial compression. That is, the fibers within a lamina that are subjected to axial compression have the propensity to change their alignment. This change is dictated by the local multiaxial stress state and the local shear stiffness of the matrix. As the local shear strains degrade the matrix shear stiffness, the resistance to fiber rotation diminishes and slowly the fiber rotation begins to build up which in turn creates more local imperfection and local shear strain. Thus a positive feedback loop is established between these two competing events. A point is reached when the in-situ shear stiffness is not sufficient to prevent the additional fiber rotation. This point usually coincides with the peak load in an axial material response curve. Beyond this point, the matrix is unable to resist any fiber rotation and the fibers rotate aligning themselves in localized deformed bands, commonly described as 'kink bands'16 (Figure 4(a)).

Consider a fiber reinforced lamina under a generalized load state as indicated in Figure 4(c). A band of misaligned fibers is shown sandwiched between two regions where the fibers are nominally straight and aligned. This equilibrium configuration is conceptualized from images of kink bands captured experimentally (Figure 4(a)). In these real kink bands, there is a conical region where fiber bending is dominant and this regions is sandwiched between the regions where there is dominant shearing (inside the band) and the farfield aligned region where there is uniform deformation (Figure 4(b)). Owing to the fiber bending dominant region, the kink band boundary inclination $\left(\beta+\beta_{0}\right)$ has the flexibility of changing during the loading, i.e., there is continuous exchange of material between the fiber bending dominant region and the shearing dominant region. In the idealization presented here, the fiber bending dominant region is disregarded and the shearing dominant region is assumed to be held in equilibrium between the two nominally aligned regions.

A reference frame $x-y$ can be defined such that the $x$-direction is parallel to the nominal fiber direction in the lamina and the $y$-direction is normal to it. In subsequent discussions, the $x-y$ reference frame will be termed as the 'global' frame. The misaligned band of fibers, in the current configuration, is defined through two angles, $\hat{\phi}=\phi+\phi_{0}$ and $\hat{\beta}=\beta+\beta_{0}$. The angles $\left(\phi_{0}, \beta_{0}\right)$ are constants and the angles $\phi$ and $\beta$ are variables that may change as a function of current far-field stress state. The reference frame, 1-2, is defined in the unstressed initial configuration of the misaligned lamina. Fibers inside the misaligned band are parallel to the ' 1 ' direction in the initial state. A current reference frame 1 ' -2 ' is defined where ' 1 ' ' is always parallel to the current fiber direction inside the band, thus the 1-axis rotates to 1 ' -axis during loading. The ' 2 ' and ' 2 ', directions are always orthogonal to the ' 1 ' and ' 1 ', directions respectively. The $1-2$ frame will be defined as the 'local' frame and the $1^{\prime}-2$ ' system will be termed the instantaneous frame. Initially, when the matrix retains most of its in-situ shear stiffness, the 1-2 and $1^{\prime}-2^{\prime}$ axes systems will be nearly co-incident. As the matrix looses its in-situ shear stiffness, local shear strain, $\gamma_{12}$, will start to rise rapidly. It can be shown that the angle between the 1 -axis and the $1^{\prime}$-axis, given by $\phi$ and $\gamma_{12}$, for small strains, are related by

$$
\gamma_{12}=\gamma_{12}^{\infty}+\phi-\beta
$$

Here, $\gamma_{12}^{\infty}$ is the contribution from the externally applied shear loading, if any. For a uniaxial compression loading and assuming $\beta=\beta_{0}=0$, we arrive at,

$$
\gamma_{12} \approx \phi
$$

\section{B. Numerical Implementation via the Finite Element (FE) Method}

The material behavior outlined in the previous subsection is modeled in the numerical domain using the commercially available FE package ABAQUS. ABAQUS has the capability of integrating user defined material behavior with its existing element library through user defined material subroutine, UMAT. ${ }^{17}$ This subroutine is called at each material point for which the constitutive law is defined through the user defined option. A UMAT receives from the solver in ABAQUS, the stresses and strains from the previous loading step, the increment of strain in the current loading step and various other parameters. After calculations, the UMAT returns to the solver the updated stresses and internal state variables, if any, and the incremental tangent stiffness matrix, $\partial \sigma_{i j} / \partial \epsilon_{i j}$. Here it is pertinent to relate the three reference frames described in the 
previous subsection to the finite element solver reference systems. The $x-y$ reference frame is the 'global' or 'laminate' frame. The master geometry of the numerical domain is defined in this system. We will also use this frame to define external loading. The $1-2$ reference frame coincides with the 'local' lamina orientation, without any loss in its significance. For the present problem, $0^{0}, 90^{\circ}$ and $\pm 45^{\circ}$ are the angles (denoted by $\theta$ in this subsection) associated for both panels studied. The solver in ABAQUS passes variables to a UMAT in this coordinate frame. The $1^{\prime}-2^{\prime}$ reference frame is the 'instantaneous' frame, with the $1^{\prime}$ direction coincident with the current fiber direction, and is used for computations within the UMAT. A description of the computation steps performed within the UMAT (which is also presented in Figure 5) is now given. In the n-th loading increment, the solver sends in the stresses $\sigma_{i j}^{n-1}$ in the $1-2$ coordinate frame which are related to the stresses in the $x-y$ frame via the transformation relation,

$$
\begin{aligned}
\sigma_{11}^{n-1} & =\cos ^{2} \theta \sigma_{x x}^{n-1}+\sin ^{2} \theta \sigma_{y y}^{n-1}+2 \cos \theta \sin \theta \tau_{x y}^{n-1} \\
\sigma_{22}^{n-1} & =\sin ^{2} \theta \sigma_{x x}^{n-1}+\cos ^{2} \theta \sigma_{y y}^{n-1}-2 \cos \theta \sin \theta \tau_{x y}^{n-1} \\
\tau_{12}^{n-1} & =\cos \theta \sin \theta\left(\sigma_{x x}^{n-1}-\sigma_{y y}^{n-1}\right)+\left(\cos ^{2} \theta-\sin ^{2} \theta\right) \tau_{x y}^{n-1}
\end{aligned}
$$

The strains $\epsilon_{i j}^{n-1}$ and strain increments $\mathrm{d} \epsilon_{i j}^{n}$ in the local coordinate system are also passed in from the solver. Within the UMAT, these local strain increments are added to the total strains to obtain the total strains $\epsilon_{i j}^{n}$. These strains, $\epsilon_{i j}^{n}$ are then transformed to the $1^{\prime}-2^{\prime}$ system using the angle between the local and the instantaneous frames, $\phi^{n-1}$ to obtain total strains in the instantaneous direction, $\epsilon_{i^{\prime} j^{\prime}}^{n}$. These strains, $\epsilon_{i^{\prime} j^{\prime}}^{n}$ are used in Eq. (15) to solve for the thermodynamic damage variable $S_{r}$. If the $S_{r}$ value thus obtained satisfies Eq. (13), then the material point accumulates damage and the lamina in situ moduli $E_{22}$ and $G_{12}$ are degraded according to the input data provided. If $S_{r}$ does not satisfy Eq. (13), then the in situ moduli are not changed from their previous values. This ensures that the material point never 'heals' and the moduli always degrade monotonically. Subsequently, the material secant constitutive matrix, $Q_{i^{\prime} j^{\prime}}^{n}$ is computed using the in situ moduli, $E_{11}, \nu_{12}, E_{22}$ and $G_{12}$. According to the present modeling scheme, $E_{11}$ and $\nu_{12}$ are not affected by $S_{r}$. Thus they retain their values at the undamaged state throughout the analysis. Next, the stresses $\sigma_{i^{\prime} j^{\prime}}^{n}$ and the material incremental constitutive matrix, $\partial \sigma_{i^{\prime} j^{\prime}} / \partial \epsilon_{i^{\prime} j^{\prime}}$ are computed. When the increment of shear strain is small, then the instantaneous fiber rotation can be equated to the change in shear strain $\mathrm{d} \gamma_{1^{\prime} 2^{\prime}}^{n} \cdot{ }^{13}$ From the constitutive relation one can also write,

$$
\gamma_{1^{\prime} 2^{\prime}}^{n}=S_{66}^{n} \tau_{1^{\prime} 2^{\prime}}^{n}
$$

where, $S_{66}=1 / G_{12}$. Taking differentials on both sides of Eq. 20,

$$
d \gamma_{1^{\prime} 2^{\prime}}^{n}=S_{66}^{n} d \tau_{1^{\prime} 2^{\prime}}^{n}+d S_{66}^{n} \tau_{1^{\prime} 2^{\prime}}^{n}
$$

Equation (21) provides an expression for the change in angle $\mathrm{d} \phi^{n}$. This change is added to the fiber angle value of the previous step to obtain the current fiber angle $\phi^{n}$.

$$
\phi^{n}=\phi^{n-1}+d \phi^{n}
$$

This angle is used in the current increment, to transform the stresses and the material incremental constitutive matrix computed in the $1^{\prime}-2^{\prime}$ frame to the $1-2$ frame, to return to the solver in ABAQUS. In the absence of damage (or when the damage is small) the angle $\phi^{n}$ will be small. But with the accumulation of damage, $\phi^{n}$ starts to increase leading to local fiber direction instability.

The steps outlined in this section are repeated at each loading increment until the analyses are completed.

\section{Details of the Experimental Studies}

\section{A. Stitched Double Notched Panels (DNPs)}

Multidirectional composite laminated specimens used in the present experimental study are cut from a master laminate which was obtained from NASA Langley Research Center and is similar to the panels 
used by McGowan et al. ${ }^{18}$ The master laminate is fabricated from Hercules Inc. AS4/3501-6 graphiteepoxy materials. It is stitched through the thickness and parallel to the principal fiber direction using E.I. Dupont de Nemours Inc. Kevlar ${ }^{(R)}$ thread. The master laminate is made of ten prefabricated 'stacks' of dry preknitted lamina each of which is $1.397 \mathrm{~mm}$ thick and has seven layers of unidirectional plies with a stacking sequence of $[ \pm 45 / 0 / 90 / 0 / \mp 45]$. The $0^{0}$ layers are parallel to the stitching direction. The resulting laminate is then infused with resin by using resin film infusion technique. Cured thicknesses of individual $\pm 45,0$, and 90 -deg layers are given in Table 1 .

Schematics of an experimental specimen is shown in Figure 2. Each specimen has overall dimensions of $210 \mathrm{~mm} \times 210 \mathrm{~mm} \times 15 \mathrm{~mm}$. Two $52.5 \mathrm{~mm}$ deep notches with a notch tip radius of $15 \mathrm{~mm}$ are cut on opposite side of each specimen. These notches create a localized high stress region which induces failure initiation and provides a predefined location for probing and obtaining diagnostics of damage initiation. Multiple DNPs have been studied. For convenience, the specimen face with thicker visible stitches is marked as the 'top' surface. Figure 1 shows the top surface of a typical specimen. A water-jet cutting procedure is used to cut the specimens from the master laminate. All four sides of each specimen are ground with a fine grit grinding wheel after cutting to ensure parallelism between opposite sides.

The unnotched sides of the specimens are used as loading edges. Each loading edge is slotted in end supports and puttied using Devcon steel putty to end caps that are made from hardened steel. This way, a 'clamped' boundary condition is obtained at each loading edge. Notched sides are slotted into anti-buckling guides. A schematic of the boundary conditions is shown in Figure 2. Experiments are carried out in an MTS machine with a crosshead displacement rate of $0.01 \mathrm{~mm} / \mathrm{s}$ and an axial load capacity of $500 \mathrm{KN}(110,000$ lbf). Back to back strain gages are used at the center of each test specimen to check for specimen bending. Six quarter bridge strain gages are placed along the line joining the notch tips on the 'top' surface of each specimen to monitor strains during loading. These locations are shown in Figure 1. Figure 6 shows a typical specimen just prior to an experiment. The bottom surface of the specimen is visible with two strain-gages which check for out-of-plane bending during the experiment. A $500 \mathrm{KN}$ capacity load-cell placed in the MTS actuator is used to measure the compressive load throughout the loading history.

\section{B. Flat Shear Panel (FSP)}

Rouse $^{7}$ performed experimental studies on flat unstiffened shear panels with and without a cutout. The effects of the material system and the laminate lay-up on the failure characteristics of the panels were studied. Figure 7 shows a typical experimental specimen from his study with a cut-out. In the present work, experimental results of a graphite-epoxy material system will be compared with simulations. The graphite/epoxy specimens were made from unidirectional tapes of Hercules Inc AS4 graphite fibers and thermosetting resin 3502. The specimens had a lay-up of $\left[ \pm 45 / 0_{2}\right]_{3 S}$ with 24 plies. Nominal thickness of each ply was $0.1397 \mathrm{~mm}$. Overall dimensions of the specimens were, $445 \mathrm{~mm}$ x $445 \mathrm{~mm} \times 3.3528 \mathrm{~mm}$. Edges of the specimen were stiffened with steel reinforcements bonded to the specimen with room temperature adhesives which produced a gage section of $305 \mathrm{~mm}$ square. A circular cut-out of $25.4 \mathrm{~mm}$ diameter was drilled at the center using diamond impregnated core drills. Tensile loading was applied to the specimen using a picture frame loading apparatus in a 1.33 MN hydraulic test machine as shown in Figure 7. Figure 8 describes the schematic of the applied load and the axis system used in testing. The global X-axis is perpendicular to the applied loading direction and the global Y-axis is parallel to the loading direction. The $45^{0}$ layers on the top surface were parallel to the applied loading. Strain gage readings from the top and bottom surfaces of the panel were recorded at a location $12.7 \mathrm{~mm}$ below the cutout along the loading diagonal (location X). Linear variable displacement transducers were used to measure the out of plane displacement at the edge of the cut-out and Moiré-fringe techniques were used to measure the complete out-of-plane deformation field of the specimen. 


\section{Numerical Simulations}

The predictive capabilities of the present PFA methodology is assessed by simulating the experimental results of the panels described in the previous section. Test sections of the experimental geometries (both the DNP and the FSP) were discreetized using the shear deformable three noded (S3) and four noded (S4/S4R) shell elements available in ABAQUS (Figure 9). Truss elements (T3D2) were used to simulate the picture frame loading frame of the FSP. A summary of the nodal and elemental data are presented in Table 2. For both the panels, static analyses were performed using displacement control loading. Linear eigenvalue buckling analyses were also performed to generate perturbations in the form of the linear eigen modes to be used in subsequent response analyses. Geometric nonlinearity is included in the response analysis through the RIKS $^{19}$ option available in ABAQUS.

Elastic material properties for the AS4/3501- $6^{20}$ and AS4 $/ 3502^{7}$ material systems are described in Table 1 and Table 3 respectively. Nonlinear shear stress-strain curves for these material systems are shown in Figure 10. A material point is denoted as 'damaged' when it reaches the end of the input stress-strain curve. For the present analysis, this refers to a $55 \%$ degradation in the in situ shear modulus, $G_{12}$. Complete material constitutive behaviors are modeled via the user material subroutine option of ABAQUS. Section lay-up of the laminates are defined using the *SHELL SECTION, COMPOSITE option available for shell elements. Thickness effects are incorporated by using multiple integration points through the thickness.

Boundary conditions for the panels are applied at nodal positions. For the DNPs, nodes corresponding to the top grip location are constrained from having any motion. Nodes occupying the bottom grip location are constrained to move only in the 1-2 plane. Rows of nodes corresponding to the knife edge support locations are constrained from moving out-of-plane. Loading applied by the MTS cross-head is simulated by specifying displacement in the 2-direction for nodes at the bottom grip location. For the flat shear panel with a cutout, effects of the steel reinforcements were modeled via rigid truss elements at the edges of the specimen. Tensile loading is simulated by specifying displacements in X- and Y- direction at point C. Equal displacements are specified such that the resultant motion is along the diagonal AC.

Geometric and material perturbations are both used in the DNP analysis. An out-of-plane perturbation equal to $0.001 \%$ of the laminate thickness is provided using the lowermost global buckling mode (obtained from a linear eigenvalue buckling analysis). In-plane geometric perturbation is provided using a negative shear loading on the bottom grip such that the bottom grip is offset by a predetermined amount with respect to the vertical (Y-direction). An offset angle of $2^{0}$ was used in the analysis. Material axis system of the axial layers ( $90^{0}$ layers) are offset from the Y-direction by $2^{0}$ in addition to the in-plane geometric perturbation. The geometric and the material axis perturbations are defined such that they act in harmony. For the FSP, only an out-of-plane geometric perturbation is used. The lowermost global buckling mode shape, obtained from a linear eigen value buckling analysis, is used for this purpose. Magnitude of the largest perturbation is $0.3 \%$ of the total laminate thickness. It is to be noted that material imperfections (misalignment of fibers within a layer) are usually on the order of $1^{0}-2^{0}$ for pre-preg based laminates.

\section{A. Results for the Stitched Panel}

Results from the DNP tests and the FE simulation are presented and salient features of compressive damage diagnostics obtained from the specimens are discussed in the present section. Experimental results are discussed first followed by the comparison with the FE simulation results. Strain readings from the back to back gages at the center of the specimen showed insignificant amount of bending being present. This was true for all three specimens examined. However, it is likely that the panels had initial geometric imperfection shapes unlike that of the lowest eigenmode shapes assumed here in the analysis to follow. This is evident in the back to back strain gage readings from the right and left notch tip areas that show differences between them suggesting that the panel deformation also shows a 'skewed $\mathrm{x}$-wise' bending, unlike the first elastic eigenmode shape.

A linear elastic FE study predicted that the notch tip compressive strain in the loading direction will

9 of 38

American Institute of Aeronautics and Astronautics Paper 2005-2368 
exceed 0.01 (10000 micro-strain) at a global reaction force of $220 \mathrm{KN}$ (Table 4). This value of axial strain is typical for fiber kinking for the present class of materials. ${ }^{14,21}$ Figure 11 presents the membrane strains (obtained from the back-to-back center gages shown in Figure 1) plotted against the reaction forces. Comparison with the finite element analysis prediction shows that the overall panel response remains largely linear during loading. Nonlinearity in the global response sets in beyond $200 \mathrm{KN}$ which is near the predicted load level for fiber kinking near the notch tip. The resultant load - displacement $(P-\Delta)$ data from the DNP tests are plotted in Figure 12. The responses show a bilinear behavior with a distinct knee around a load of $220 \mathrm{KN}$. A slight 'kink' in the P- $\Delta$ responses near $340 \mathrm{KN}$ is also observed. There was no audible or visible indication of damage prior to this load level and the DNP specimens failed catastrophically at an external load of 370 $\mathrm{KN}$. The specimens separated in two halves during their removal from the end supports. Comparison of the catastrophic failure loads of the DNP specimens (Table 5) with the predicted linear buckling load (Table 4) shows that specimen failure did not occur due to global buckling. Instead, progressive damage accumulation in all the layers in the vicinity of each notch tip leads to a zone of 'degraded' material. These zones grow towards each other just prior to catastrophic failure and the panel undergoes an out-of-plane shear failure separating into two halves as shown in the post-experiment image of Figure 13.

Figure 13 shows the top surface of the first DNP specimen (Test-1) after it was removed from the fixture. As it can be seen, damage which initiated near the notches grew suddenly across the specimen above the center stitch. On the bottom surface, a damage zone propagated across the specimen below the center stitch (Figure 14(a)). Presence of the center stitch seems to prevent damage initiation exactly at the notch tip. It is also seen that a view of the through thickness damage is inclined at an angle to the loading axis rather than being perpendicular to it (Figure 14(b)).

In view of these observations from the panels studied, a third DNP specimen was tested such that damage would initiate in the vicinity of the notch tips but would not progress towards catastrophic failure. This specimen was loaded up to $340 \mathrm{KN}$ and subsequently unloaded. Strain gage readings from the gages near the notch tips showed nonlinearity at this load level indicating the presence of internal damage. The unloaded specimen was taken off the grips and cut along the center line of the left notch tip gage (section AA', Figure 1). Post-experiment damage diagnostics were performed using optical microscopy and Scanning Electron Microscopy (SEM). Figure 15 shows the AA' sectional view of the notch tip looking from inside the laminate. Multiple damage locations on this section are indicated with 'ABCD' close-up markers and studied with the aid of an SEM. Fiber kinking in the vicinity of the notch tip is the main source of damage (Figure 16 and Figure 17).

Load-displacement comparison

Load-vs.-load point displacement data for the DNP test specimens are compared with the FE result in Figure 12. The experimental data show regions of 'settling' in the initial stages. The FE simulation results are plotted after accounting for the settling of laboratory specimens. The overall behavior of the FE simulation is similar to the experimental observations. Initial slopes of the curves presented match quite well. Beyond $220 \mathrm{KN}$, the FE result does not show the pronounced 'knee' as seen in the experimental curves. Instead it remains bounded by the experimental responses. The FE analysis predicts a peak load of $386 \mathrm{KN}$ which is within $3 \%$ of the experimentally observed maximum load.

Strain-gage measurements

The strain readings recorded from the DNP panels are shown in Figure 18. The locations of the gages are indicated in Figure 1 and tabulated in Table 6 . All strains initially are linear with the applied load, but a careful examination shows a softening behavior as loading proceeds. This nonlinearity is progressive and is indicative of damage accumulation in the panel. As expected, the gages closest to the notch tips record the highest values and the intensity decreases with distance away from the notch tips. The back to back notch tip gages show bending and also differ between the right notch and left notch (Figure 18(a),(c) and Figure 18(b),(d)), suggesting a width wise skewed (in the x-direction) bending during the deformation. An acceleration of the notch tip strain nonlinearity is observed at $300 \mathrm{KN}$ (gage 6 in Figure 18(d)) and the gage is rendered malfunctional at about $310 \mathrm{KN}$. The FE results, because they are based on a panel with a very slight out-of-plane geometric imperfection in the form of the smallest elastic eigen mode, does

10 of 38

American Institute of Aeronautics and Astronautics Paper 2005-2368 
not show any differences in the back to back notch tip readings. The FE data is also 'stiffer' as expected, indicating that the panel properties are most likely underestimated. However, the trends in all the strains captured by the FE simulation are correct with respect to softening nonlinearity and with respect to the acceleration of the non-linearity as loading proceeds. Indeed, all the FE strain readings become near vertical at approximately $384 \mathrm{KN}$, indicative of the softening induced localized instability experienced by the panel. An examination of the strain readings appears to indicate that the strain predictions of the FE simulations are slightly shifted. That is, if all the panel strain readings are correlated with the FE strains at locations that are further away from the notch, then the readings show a remarkable agreement. This suggests that a 'scaling' of the material properties and proper accounting for the initial geometric imperfections should bring agreement much closer. It must be noted that the FE simulations are based on classical lamination theory ideas (strictly 2D) which neglect possible 3D effects that can also contribute to the discrepancies. A study that examines the effects of material property uncertainty was carried out in a limited sense for the FSP (see later). A similar study for the DNP is currently under progress.

Damage contours at various load levels

Contours of the damage variable $S_{r}$ are presented at increasing load levels in Figure 19 through Figure 21. Results are presented for the top four layers, $\pm 45^{0}, 0^{0}$ and $90^{\circ}$ layers, where the layer orientation is given with respect to the $\mathrm{x}$-direction. A color closer to red indicates a higher level of degradation compared to a color closer to blue which indicates zero or minimal damage. The dark areas in these maps correspond to shear stiffness loss of $55 \%$ or more. In the following discussion, damage state or level of degradation is used interchangeably with the loss of shear stiffness. It is readily observable that different layers are at different levels of degradation at a given global reaction force. It is also noticeable that the $90^{0}$ layer which is under global axial compression, shows the least amount of damage whereas the $\pm 45^{0}$ layers show the most damage. The axial layer damage, suddenly starts to accelerate and grow near and beyond the peak load. The $\pm 45^{0}$ layers make up nearly $60 \%$ of the laminate, thus the global stiffness of the system is greatly affected by the degradation of these layers. In a through-thickness view at the notch tips, at a load of $350 \mathrm{KN}$ load, only $14 \%$ of the layers have substantial shear stiffness remaining and the rest have lost $55 \%$ or more of their shear stiffnesses. Thus the laminate weakens in both inplane and out-of-plane directions. Damage zones grow a little offset from the notch tips in the $90^{\circ}$ layer and converges towards the notch tips with increasing load. In the $\pm 45^{0}$ layers, damage nucleates from the notch tips in a small region. Then the two separate damage zones propagate towards each other along the center line of the specimen, finally establishing a region of uniformly damaged material between the two notches (the continuous red band in Figure 21). Evidence of this phenomenon is readily visible in the damage contours of the $0^{0}$ layer. The contours do not appear to change much between the peak load $(386 \mathrm{KN})$ and the $384 \mathrm{KN}$ load levels. Under closer inspection, the distance between the fronts of the advancing damage zones are smaller at $384 \mathrm{KN}$ compared to $386 \mathrm{KN}$ indicating continuous damage growth. This damage growth coupled with the strain gage readings becoming near vertical signals the onset of catastrophic failure.

\section{B. Results for the flat unstiffened shear panel}

Simulation results for the FSP are compared with the experimental results obtained by Rouse. ${ }^{7}$ Experimental observations for the FSP reported that the panel failed deep into the post-buckling regime. Delamination zones and tension cracks developed at the edges of the cut-out and lead to the failure of the specimen. Two types of data are reported from the experiment, namely load-vs.-load point displacement and load-vs.-surface strain variation. Similar data sets are generated from FE simulations and compared with the experimentally reported data.

Three different FE cases are reported in the following discussions. Finite element analysis with the elastic properties as reported by Rouse ${ }^{7}$ is denoted by 'case 1'. Case 2 denotes analysis with elastic moduli values $110 \%$ of those reported by Rouse. ${ }^{7}$ A third analysis, using an explicit tensile failure criterion was performed and those results are reported under 'case 3'.

Load-displacement comparison

11 of 38

American Institute of Aeronautics and Astronautics Paper 2005-2368 
Load $(\mathrm{P})$ and load point displacement $(\Delta)$ data from the experiment and the simulations are plotted in Figure 22. The experimental buckling load for the specimen was reported to be $112 \mathrm{KN}$. A linear eigenvalue buckling analysis of the FSP geometry produced a buckling load of $113 \mathrm{KN}$ in the simulation.

Strain-gage measurements

Figure 23 shows the variation of reaction force $\mathrm{P}$ with strains $\epsilon_{22}$ (perpendicular to the fiber direction) measured on the top and bottom surfaces of the panel at a station $12.7 \mathrm{~mm}$ below the cutout edge along the loading diagonal (Figure 8). It can be seen that the present analysis is able to capture the experimentally observed phenomena of bifurcation and subsequent strain evolutions on the top and bottom surfaces. They also show a softer behavior compared to the experimental observation, which is due to the incorporation of continuous material damage. Exact data for the stiffness degradation of the AS4/3502 system used in the FSP experiments were not available. Hence, the degradation behavior (i.e., the $S_{r}$-vs.-normalized stiffness relations) of the AS4/3501-6 system was used as an approximation. The results show that such a degradation behavior provides a 'lower' bound for the FSP panel (FE, case 1 in the figures). The 'FE, case 2 ' analysis was performed by increasing the elastic constants of the FSP material by $10 \%$ and keeping the degradation behavior unchanged. This approach led to an 'upper' bound of the FSP material. The actual specimen material properties lie somewhere in between these two limits. The $\epsilon_{22}-\mathrm{P}$ behavior suggests that the degradation law (form of the $S_{r}$-vs.-normalized $G_{12}$ curve) has a lesser effect on the system response of these FSPs than the initial undamaged moduli.

Damage maps of various layers

Various locations are marked on Figure 22 which correspond to the various damage events occurring in the laminate. Table 7 shows the loads associated with these events. Contours of damage variable $S_{r}$ for each of these events are shown for the top three layers $\left( \pm 45^{0}\right.$ and $0^{0}$ layers $)$ and the bottom layer $\left(45^{0}\right.$ layer) in Figure 24 through Figure 27. The first set of contours (Figure 24) show the material state just prior to global buckling. It can be seen that damage occurs in limited areas in various plies. Global FSP buckling coupled with other failure events, accelerates material degradation (Figure 25- Figure 27). The damage is seen to nucleate in areas surrounding the edge of the cut-out. Propagation of the damage zones are mainly perpendicular $\left(-45^{0}\right.$ and $0^{0}$ plies) and parallel (bottom $45^{0}$ ply) to the loading direction. Beyond the global buckling event, damage accelerates in the bottom $45^{0}$ ply compared to the other layers. At a global load of $118 \mathrm{KN}$, the contours of damage in various layers are shown in Figure 25. During subsequent loading, degradation accelerates in other layers also and at $129 \mathrm{KN}$ (event B) tensile failure nucleates in the top $45^{0}$ layer. Figure 28 shows the contours of the damage variable $S_{r}$ near the cut-out at event B. It is readily observable that the $-45^{0}$ layer and the $0^{0}$ layer near the cut-out (at locations perpendicular to the external loading) are at a higher level of damage compared to the outermost layers. Thus these locations subsequently loose support for the outermost layers and other damage modes appear. Results from 'FE, case 3' incorporates contours of a variable which is monitored during the analysis to indicate when the local tensile axial stress exceeds the tensile strength of the material $(1378 \mathrm{KN}) .^{8}$ The initiation of tensile failure occurs in the topmost layer followed by the tensile failure in the bottom layer (event C) and coincides with the inner layers loosing $55 \%$ of their in situ shear stiffness at those locations. Figure 29 shows the growth of tensile damage which is perpendicular to start with but slowly aligns itself parallel to the loading direction in the topmost layer. As the tensile failure zone extends from its nucleation site, the strain in the topmost layer is seen to deviate from the ' $\mathrm{FE}$, case 1' results (Figure 23). In the laboratory experiment, the material degradation and tensile failure could have propagated in a violent manner leading to a sudden catastrophic failure. The contours of damage variables clearly show that the present analysis is able to capture the different rates of damage progression in the top and the bottom layers.

Out-of-plane displacement data

Figure 30 shows the out-of-plane displacement(w) at the cut-out boundary along the loading diagonal (Figure 8) plotted against the global reaction force P. Experimental data of w-vs.-P for the experimental FSP specimen analyzed here are not available (these results are available for FSP specimens with different material systems and laminate lay-ups). But the trends shown are similar to the experimental results reported by Rouse. $^{7}$ 


\section{Concluding Remarks}

In this paper, a failure mechanism based progressive failure analysis methodology is developed and validated against experimental data for two types of structural panels. The methodology uses the complete non-linear stress-strain relations for the lamina in shear and in transverse tension/compression as input, along with readily available lamina level elastic properties. Using only these (a minimum number) as inputs, progressive failure and damage growth in two types of structural panels, loaded under a pre-dominantly compression loading have been simulated. In particular by modeling the physics of the kink banding process responsible for limiting the lamina axial compression strength, the maximum load sustained by the panels and the subsequent damage evolution have been captured accurately. The notion of a fixed compression strength has been disbanded, instead the in-situ compression strength of a lamina is determined as a part of the solution process, and as a function of loading history. The success of the present PFA methodology is encouraging and points the way for extending the methodology to analyze other structural configurations.

\section{Acknowledgments}

The authors are grateful for financial sponsorship through NASA Langley research grant NCC-1-01050.

\section{References}

\footnotetext{
${ }^{1}$ Hashin, Z., "Failure Criteria for Unidirectional Fiber Composites," Journal of Applied Mechanics, Vol. 47, June 1980, pp. $329-334$.

${ }^{2}$ Chang, F.-K. and Lessard, L. B., "Damage Tolerance of Laminated Composites Containing an Open Hole and Subjected to Compressive Loadings: Part I-Analysis," Journal of Composite Materials, Vol. 25, January 1991, pp. 2-43.

${ }^{3}$ Schapery, R. A., "A Theory of Mechanical Behavior of Elastic Media with Growing Damage and other Changes in Structure," J. Mech. Phys. Solids, Vol. 38, No. 2, June 1990, pp. 215-253.

${ }^{4}$ Schapery, R. A. and Sicking, D. L., "On Nonlinear Constitutive Equations for Elastic and Viscoelastic Composites with Growing Damage," Mechanical Behavior of Materials, Vol. 47, June 1995, pp. 45-76.

${ }^{5}$ Oguni, K., Tan, C. Y., and Ravichandran, G., "Failure Mode Transition in Unidirectional E-Glass/Vinylester Composites under Multiaxial Compression," Journal of Composite Materials, Vol. 34, No. 24, 2000, pp. 2081-2097.

${ }^{6}$ Basu, S., Waas, A. M., and Ambur, D. R., "Compressive Failure of Fiber Composites under Multiaxial Loading," in review, 2005.

${ }^{7}$ Rouse, M., "Effect of Cutouts or Low-speed Impact Damages on the Postbuckling Behavior of Composite Plates Loaded in Shear," $31^{\text {st }}$ SDM Conference, AIAA, , No. AIAA-90-0966-CP, 1990, pp. 877-891.

${ }^{8}$ Ambur, D., Jaunky, N., Dávila, C., and Hillburger, M., "Progressive Failure Studies of Composite Panels with and without Cutouts," 42 $2^{\text {nd }}$ SDM Conference, AIAA, Seattle, , No. AIAA-2001-1182-CP, April 2001, pp. 1-11.

${ }^{9}$ Goyal, V. K., Jaunky, N., Johnson, E. R., and Ambur, D., "Intralaminar and Interlaminar Progressive Failure Analysis of Composite Panels with Circular Cutouts," 43 ${ }^{\text {rd }}$ SDM Conference, AIA A, Denver, , No. AIAA 2002-1745, 2002, pp. 1-12.

${ }^{10}$ Schapery, R. A., "Mechanical Characterization and Analysis of Inelastic Composite Laminates with Growing Damage," Mechanics of Composite Materials and Structures, Vol. AMD-100, June 1989, pp. 1-9.

${ }^{11}$ Sun, C. T. and Chen, J. L., "A Simple Flow Rule for Characterizing Nonlinear Behavior of Fiber Composites," Journal of Composite Materials, Vol. 23, October 1989, pp. 1009-1020.

${ }^{12}$ Dávila, C. G., Ambur, D. R., and McGowan, D. M., "Analytical Prediction of Damage Growth in Notched Composite Panels Loaded in Compression," Journal of Aircraft, Vol. 37, No. 5, September-October 2000, pp. 898-905.

${ }^{13}$ Schapery, R. A., "Prediction of Compressive Strength and Kink Bands in Composites using a Work Potential," International Journal of Solids and Structures, Vol. 32, No. 6/7, 1995, pp. 739-765.

${ }^{14}$ Lee, S. H. and Waas, A. M., "Compressive Response and Failure of Fiber Reinforced Unidirectional Composites," International Journal of Fracture, Vol. 100, No. 3, December 1999, pp. 275-306.

${ }^{15}$ Schapery, R. A., "Private communication," 2002.

${ }^{16}$ Evans, A. G. and Adler, W. F., "Kinking as a Mode of Structural Degradation in Carbon Fiber Composites," Acta Metallurgica, Vol. 26, 1978, pp. 725-738.

${ }^{17}$ Anon., ABAQUS User's Manual, Ver. 6.3-1, Hibbit, Karlson and Sorenson, Pawtucket, RI, 2003.

${ }^{18}$ McGowan, D. M., Dávila, C. G., and Ambur, D. R., "Damage Progression in Buckle-Resistant Notched Composite Plates Loaded in Uniaxial Compression," 42 ${ }^{\text {nd }}$ SDM Conference, AIAA, Seattle, April 2001.
}

13 of 38 
${ }^{19}$ Riks, E., "The Application of Newton's Method to the Problem of Elastic Stability," Journal of Applied MechanicsTransactions of the ASME, Vol. 39, No. 4, December 1972, pp. 1060-1065.

${ }^{20}$ Soden, P., Hinton, M. J., and Kaddour, A. S., "Lamina Properties, Lay-up Configurations and Loading Conditions for a Range of Fiber-Reinforced Composite Laminates," Composite Science and Technology, Vol. 58, 1998, pp. 1011-1022.

${ }^{21}$ Kyriakides, S., Arseculeratne, R., Perry, E. J., and Liechti, K. M., "On the Compressive Failure of Fiber Reinforced Composites," International Journal of Solids and Structures, Vol. 32, No. 6/7, 1995, pp. 689-738.

${ }^{22}$ Yerramalli, C., A Mechanism based Modeling Approach to Failure in Fiber Reinforced Composites, Ph.D. thesis, Aerospace Engineering Department, University of Michigan, Ann Arbor, 2003. 
Table 1. Properties of the different prefabricated ply orientations. ${ }^{18}$

\begin{tabular}{|c|c|c|c|}
\hline \multirow{2}{*}{ Properties } & \multicolumn{3}{|c|}{ Layer Orientation } \\
\cline { 2 - 4 } & $\pm 45^{0}$ & $0^{0}$ & $90^{0}$ \\
\hline thickness $(\mathrm{mm})$ & 0.14986 & 0.15697 & 0.16967 \\
\hline$E_{11}(\mathrm{GPa})$ & 111.0 & 113.0 & 110.0 \\
\hline$E_{22}(\mathrm{GPa})$ & 11.0 & 11.0 & 11.0 \\
\hline$G_{12}(\mathrm{GPa})$ & 5.5 & 5.5 & 5.5 \\
\hline$G_{13}(\mathrm{GPa})$ & 5.5 & 5.5 & 5.5 \\
\hline$G_{23}(\mathrm{GPa})$ & 2.8 & 2.8 & 2.8 \\
\hline$\nu_{12}$ & 0.34 & 0.34 & 0.34 \\
\hline
\end{tabular}

Table 2. Summary of nodal and elemental data for the FE analyses

\begin{tabular}{|c|c|c|}
\hline Elements & 11713 & 2699 \\
\hline S3/S4 & 11713 & 2635 \\
T3D2 & - & 64 \\
\hline Nodes & 11951 & 2697 \\
\hline Degrees of Freedom & 71706 & 16182 \\
\hline
\end{tabular}

Table 3. Properties of the different prefabricated ply orientations. ${ }^{7}$

\begin{tabular}{|c|c|}
\hline Properties & \\
\hline thickness $(\mathrm{mm})$ & 0.1397 \\
\hline$E_{11}(\mathrm{GPa})$ & 131.0 \\
\hline$E_{22}(\mathrm{GPa})$ & 13.0 \\
\hline$G_{12}(\mathrm{GPa})$ & 6.4 \\
\hline$G_{13}(\mathrm{GPa})$ & 6.4 \\
\hline$G_{23}(\mathrm{GPa})$ & 1.8 \\
\hline$\nu_{12}$ & 0.38 \\
\hline
\end{tabular}

Table 4. Predictions from a linear elastic FE analysis of the DNP geometry. Negative strains indicate compression.

\begin{tabular}{|c|c|}
\hline Event & Global Load (KN) \\
\hline Notch tip axial strain -0.010 & 220 \\
\hline Notch tip axial strain -0.015 & 330 \\
\hline Global buckling & 1300 \\
\hline
\end{tabular}


Table 5. Summary of experimental observation of the DNP specimens. Negative strains indicate compression.

\begin{tabular}{|c|c|c|}
\hline & Ultimate failure load (UFL) $(\mathrm{KN})$ & Axial strain at UFL $(\mu$-strain $)$ \\
\hline Test-1 & 372 & -6110 \\
\hline Test-2 & 375 & -5943 \\
\hline
\end{tabular}

Table 6. Summary of the experimental strain measurement stations for the DNP panel.

\begin{tabular}{|c|c|c|}
\hline $\begin{array}{c}\text { Distance from the } \\
\text { left notch tip }\end{array}$ & Test-1 & Test-2 \\
\hline Gage 1 & $5 \mathrm{~mm}$ & $2 \mathrm{~mm}$ \\
\hline Gage 1 back & - & $3 \mathrm{~mm}$ \\
\hline Gage 2 & $12 \mathrm{~mm}$ & $10 \mathrm{~mm}$ \\
\hline Gage 3 & $25 \mathrm{~mm}$ & $25 \mathrm{~mm}$ \\
\hline $\begin{array}{c}\text { Distance from the } \\
\text { right notch tip }\end{array}$ & & \\
\hline Gage 4 & $24 \mathrm{~mm}$ & $25 \mathrm{~mm}$ \\
\hline Gage 5 & $12 \mathrm{~mm}$ & $11 \mathrm{~mm}$ \\
\hline Gage 6 & $6 \mathrm{~mm}$ & $3 \mathrm{~mm}$ \\
\hline Gage 6 back & - & $3 \mathrm{~mm}$ \\
\hline
\end{tabular}

Table 7. Summary of various failure events and corresponding loads for the FSP panel.

\begin{tabular}{|c|c|}
\hline Event & Load \\
\hline Global Buckling & $107 \mathrm{KN}$ \\
\hline (A) Bottom ply $G_{12}$ degrades $55 \%$ & $118 \mathrm{KN}$ \\
\hline $\begin{array}{c}\text { (B) Top ply tensile failure } \\
\text { (Top Layer) }\end{array}$ & $129 \mathrm{KN}$ \\
\hline (C) Bottom ply tensile failure & $143 \mathrm{KN}$ \\
\hline
\end{tabular}




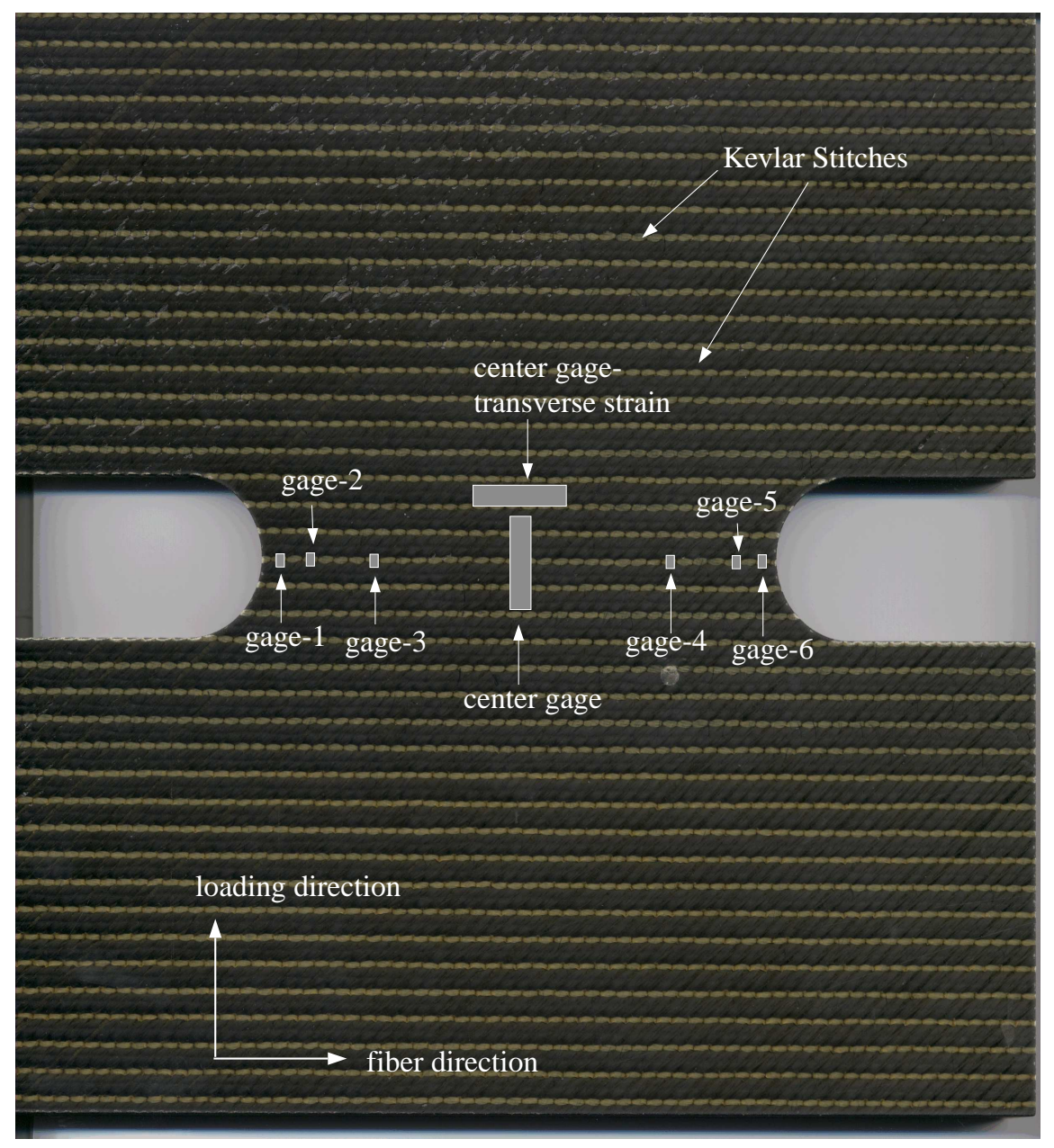

Figure 1. Top surface of a DNP specimen. Strain gage locations are shown with white boxes. 


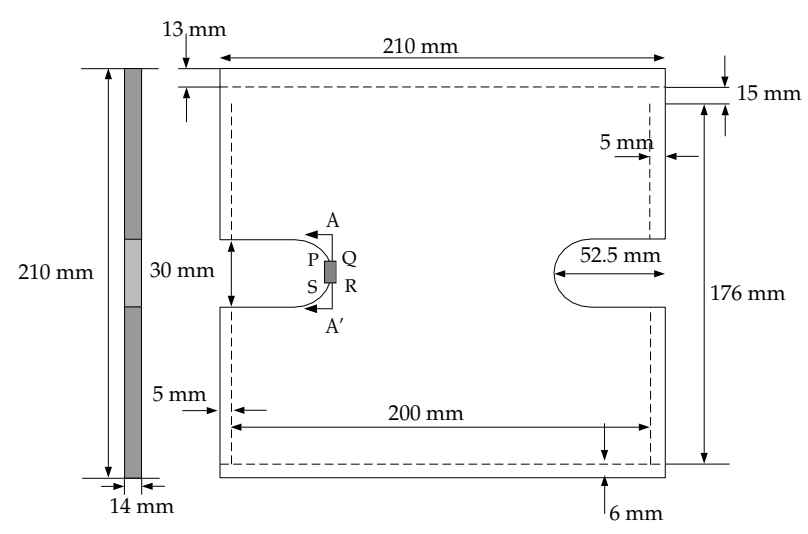

(a) Dimensions of the DNP

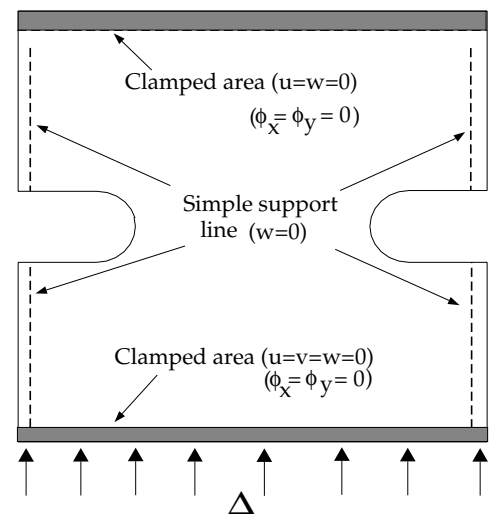

(b) Boundary conditions of the DNP

Figure 2. Schematics of the specimens and test conditions used in the present study. Section $A A^{\prime}$ is used to cut Test-3 specimen for damage diagnostics. The kinked zero-ply viewed as PQRS is shown in Figure 17.

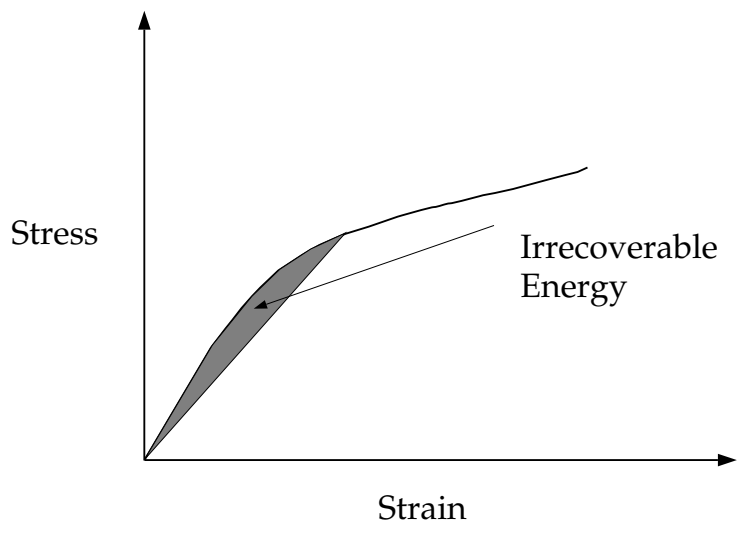

Figure 3. Definition of irrecoverable energy using a generic stress-strain curve. 


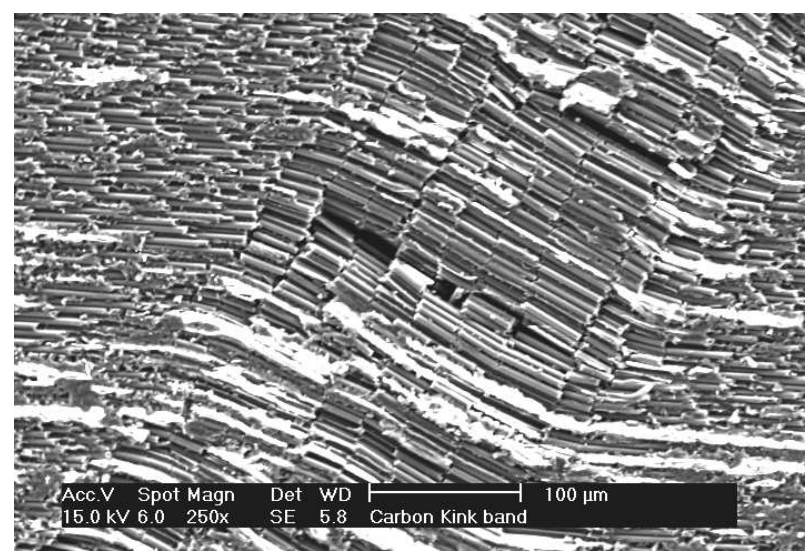

(a) Developing kink band

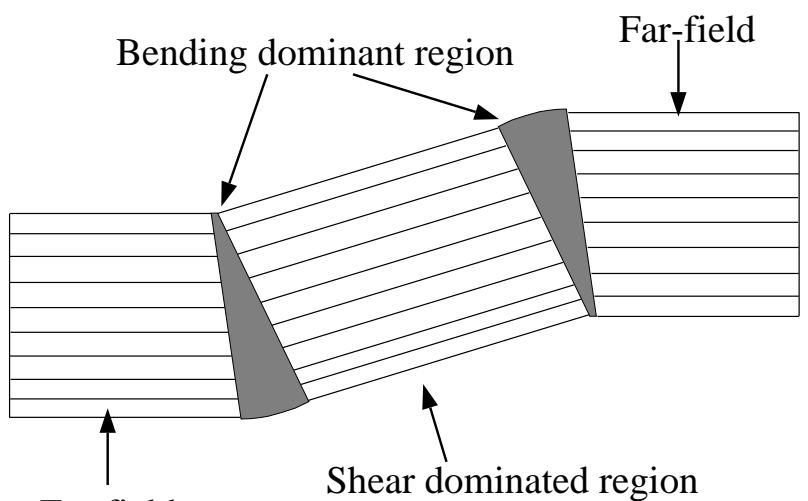

Far-field

Shear dominated region

(b) Schematic of a lamina

Figure 4. The developing kink band in Carbon composites ${ }^{22}$ and the schematic representation of such within a lamina.

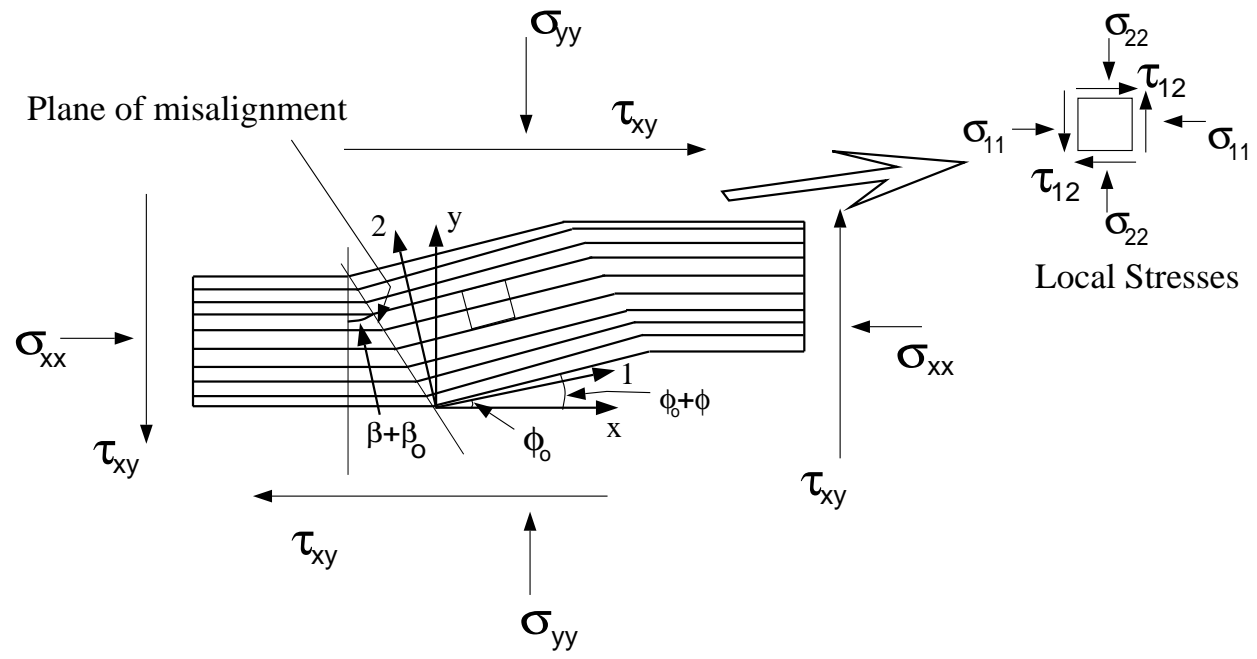

Figure 4.(c) A unidirectional lamina with a band of misaligned fibers in equilibrium under a multiaxial stress state. Inset shows the stress state inside the misaligned band. 


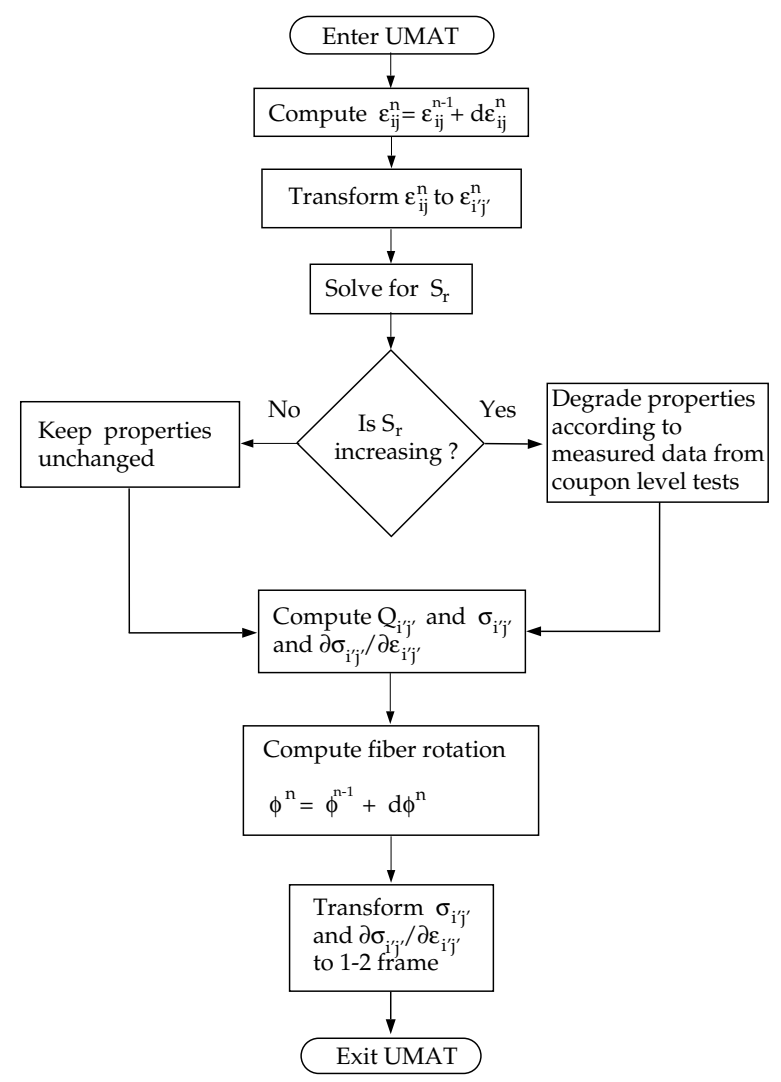

Figure 5. Flow chart of the operations performed inside a UMAT for implementing the fiber rotation and Schapery Theory ideas.

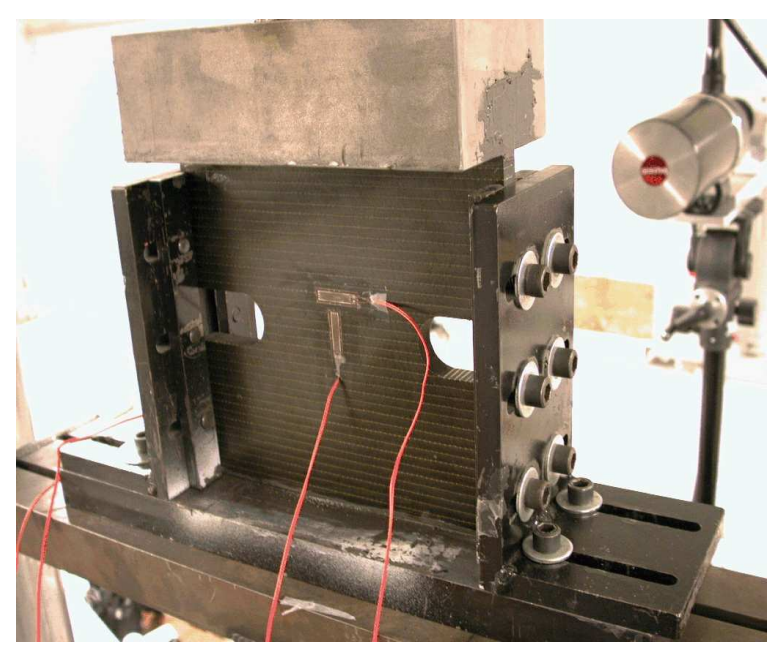

Figure 6. A DNP specimen is shown with supports and bottom surface strain gages prior to an experiment.

20 of 38

American Institute of Aeronautics and Astronautics Paper 2005-2368 


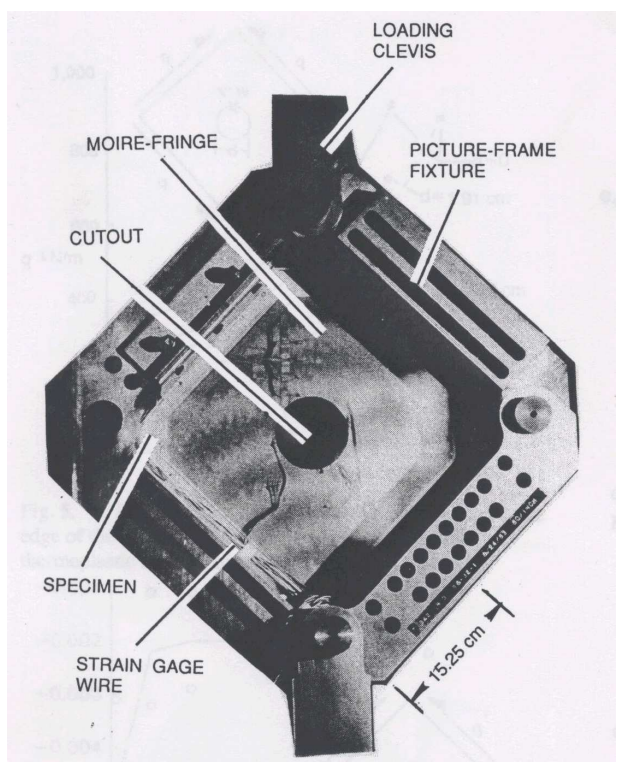

Figure 7. Typical experimental set-up for FSPs. ${ }^{7}$

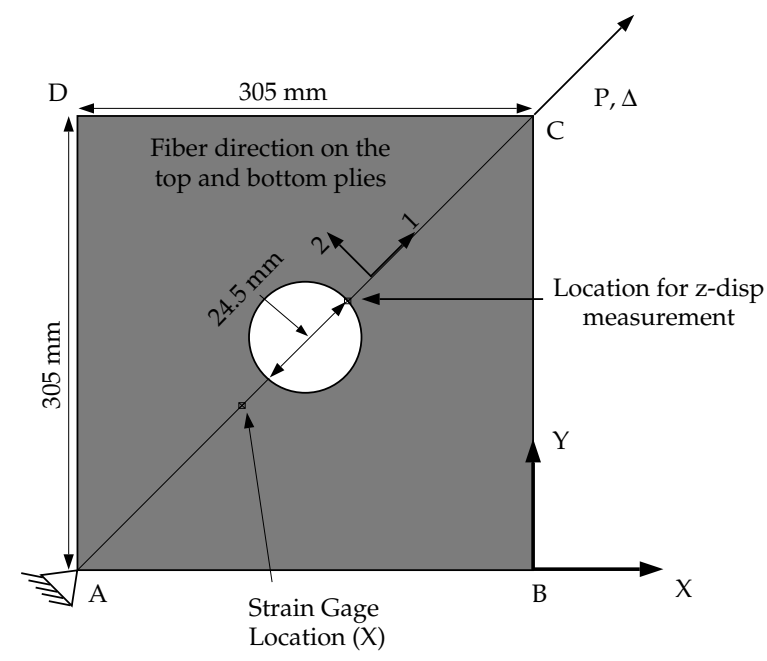

Figure 8. Schematic geometry of the FSP specimen. 


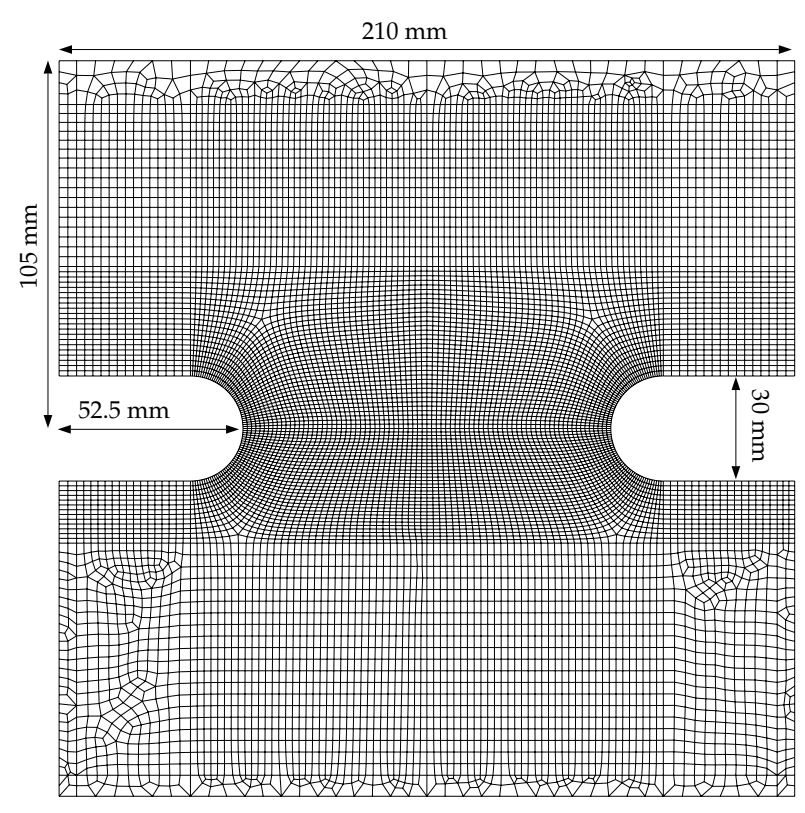

(a) DNP FE mesh

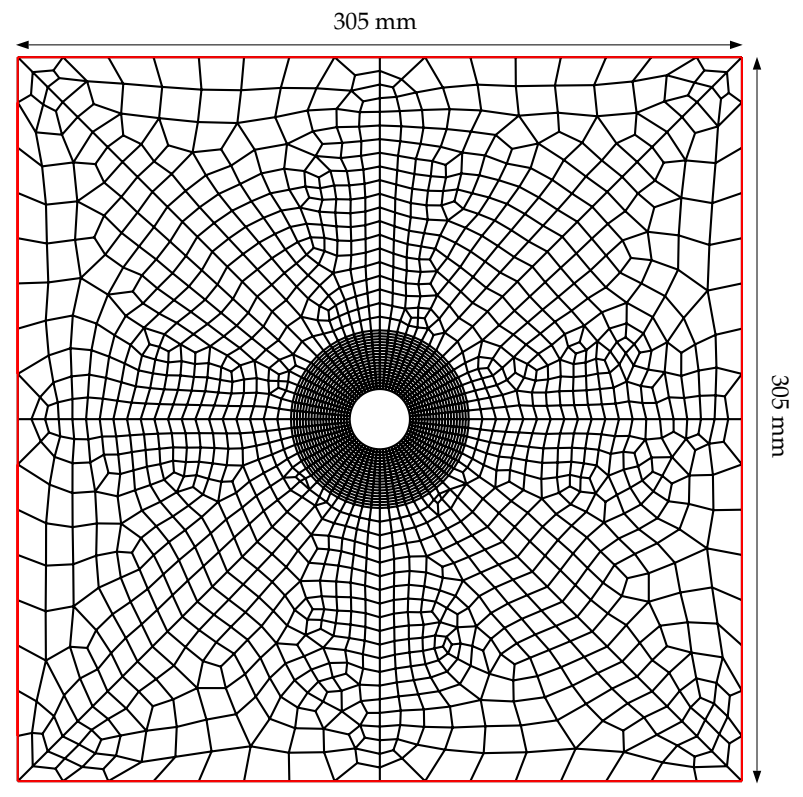

(b) FSP FE mesh

Figure 9. Finite element meshes used in the present study.

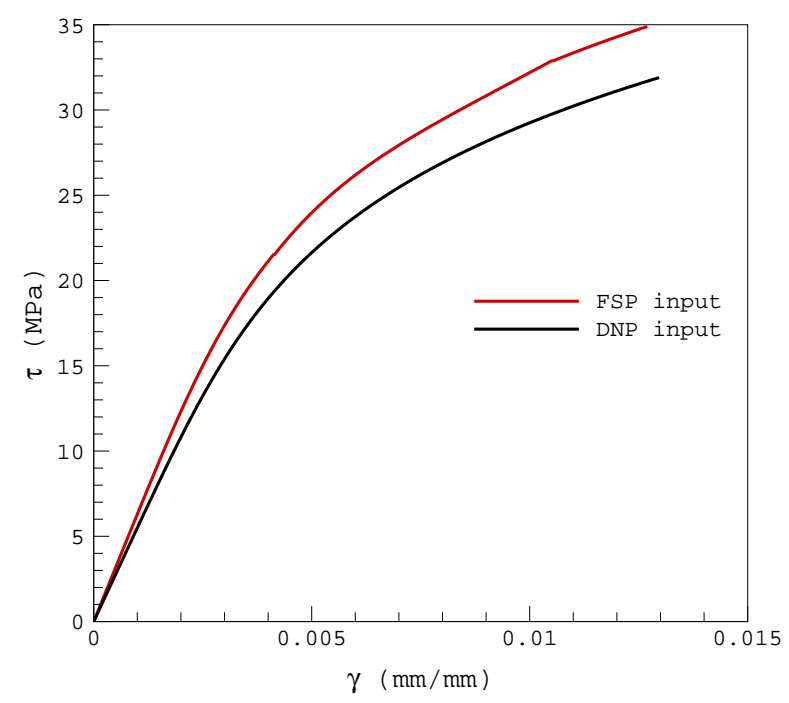

Figure 10. Material inelastic input curves used in the present study. 


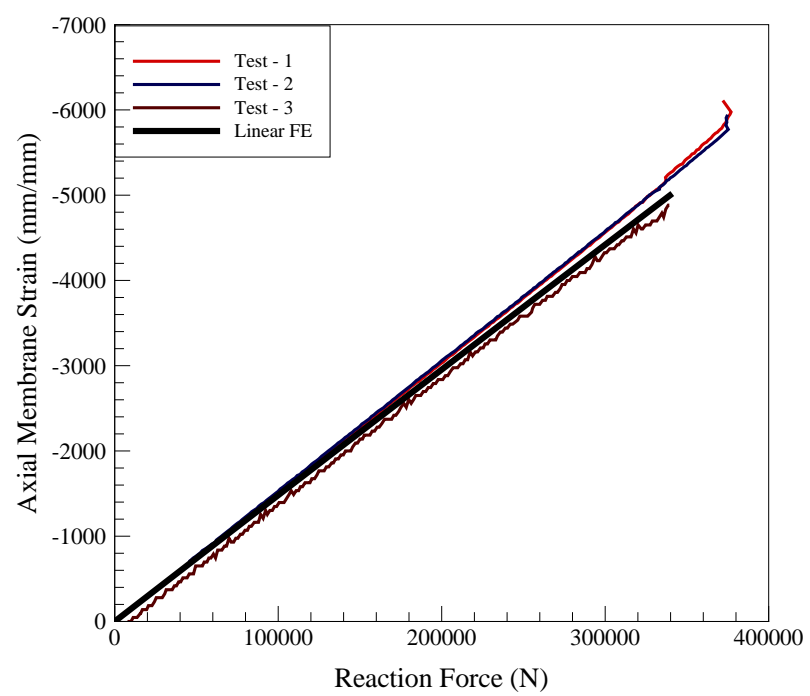

Figure 11. Membrane strain plotted against reaction force from three DNP specimens and the FE analysis. Notice the repeatability of the test results and agreement with the FE prediction.

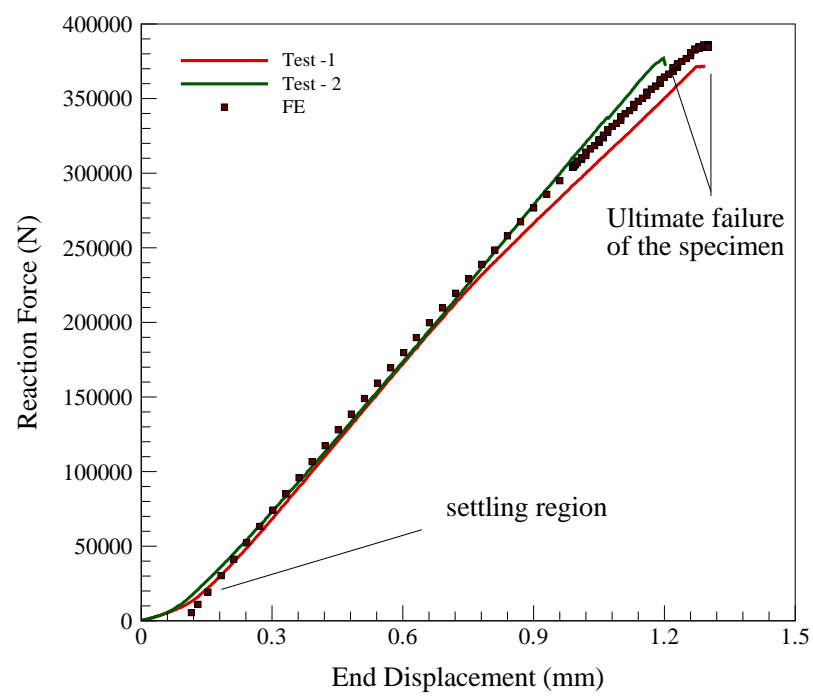

Figure 12. Load-vs.-displacement data comparison of the DNP experiments and the numerical simulation. 


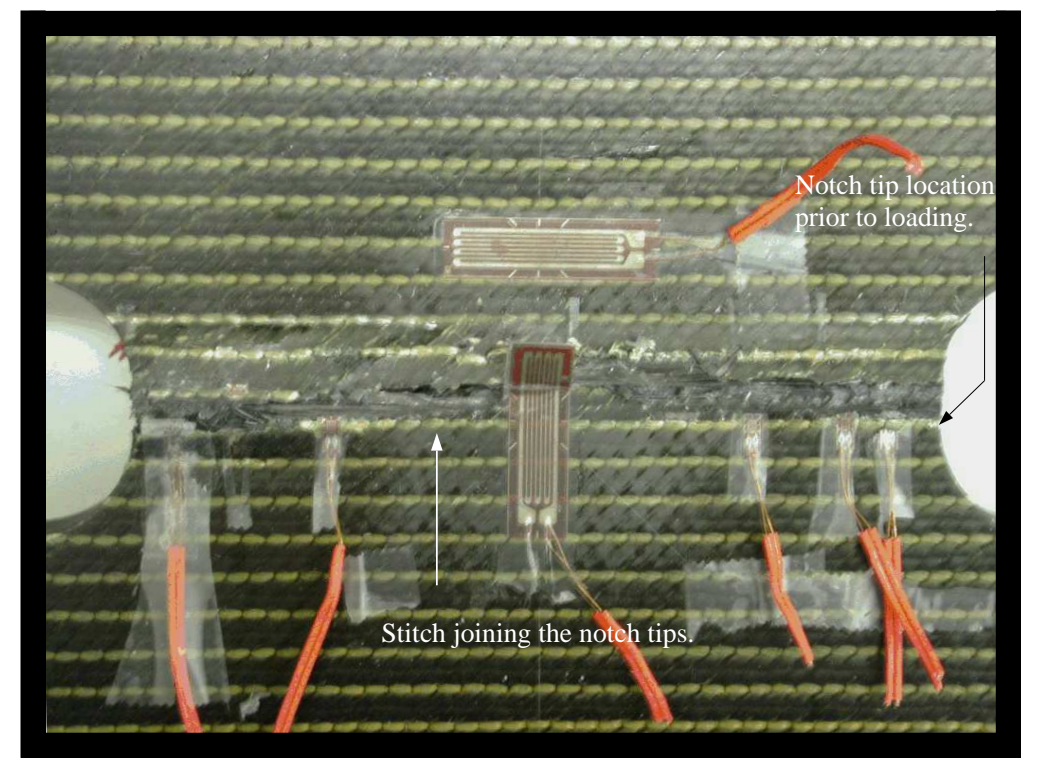

Figure 13. Top surface of a DNP specimen is shown after being loaded beyond catastrophic failure.

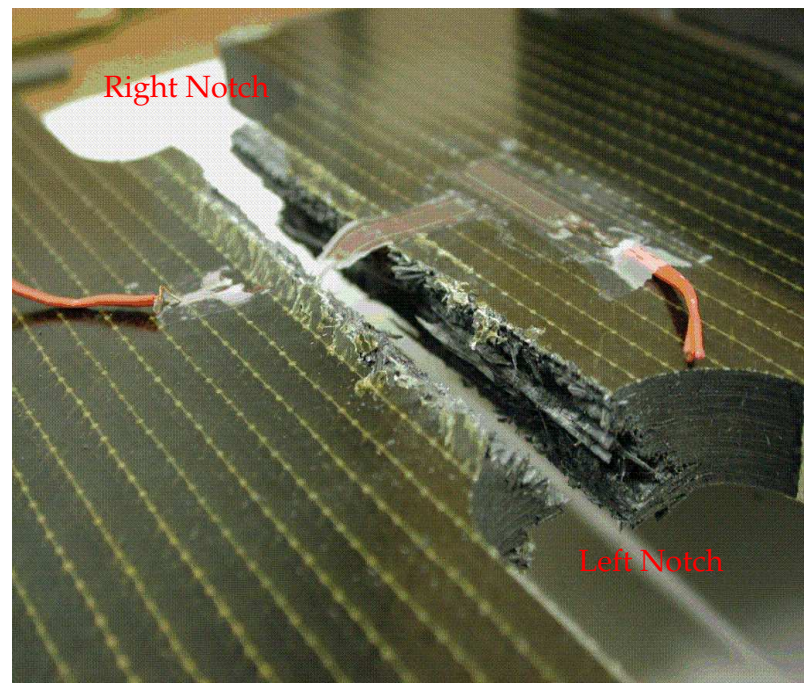

(a) Left Notch

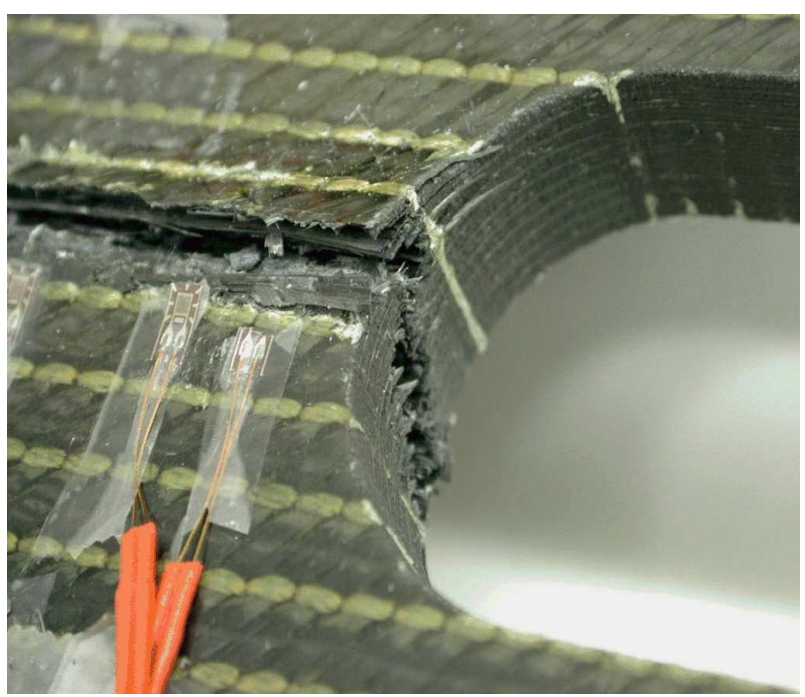

(b) Right Notch

Figure 14. Close-ups of the notches of the specimen shown in Figure 13 after experiment. 


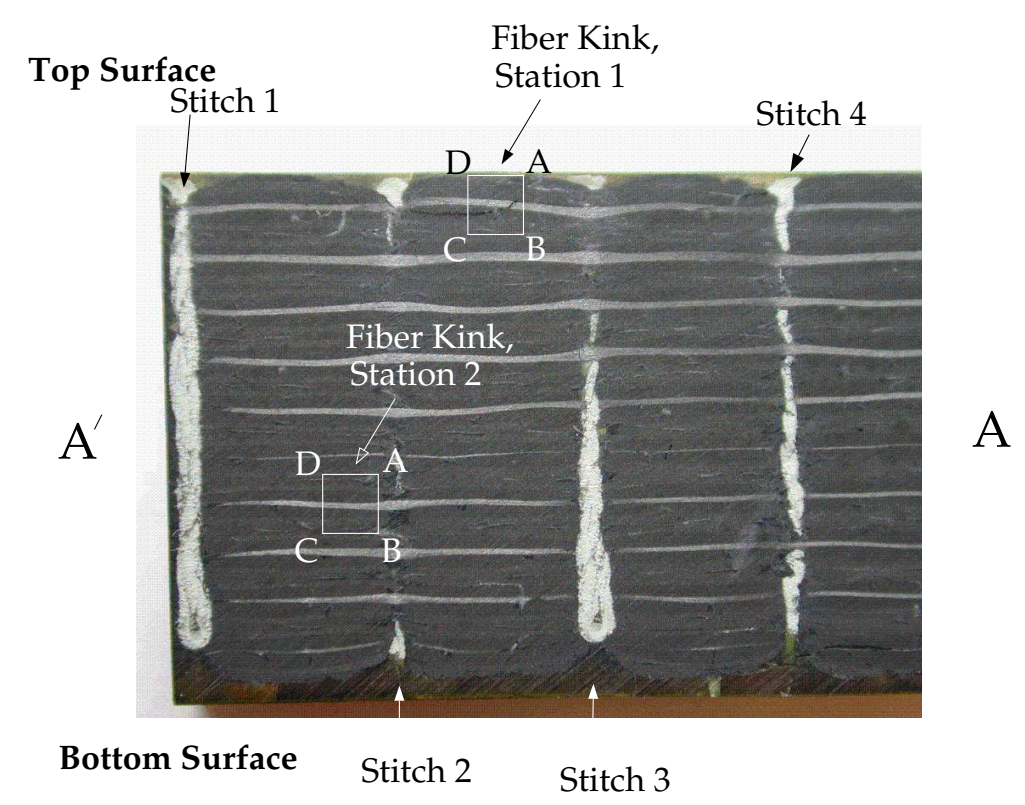

Figure 15. Cross-section view $A A^{\prime}$ (Figure 2) behind the left notch tip. Prominent damage locations are marked and the corresponding SEM pictures are shown in subsequent figures. Global loading is applied along the vertical.

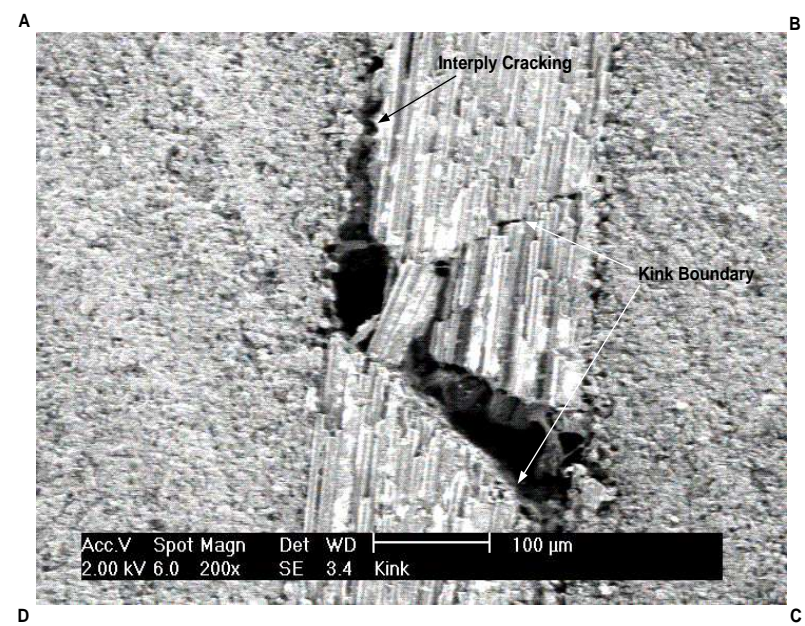

(a) Station 1

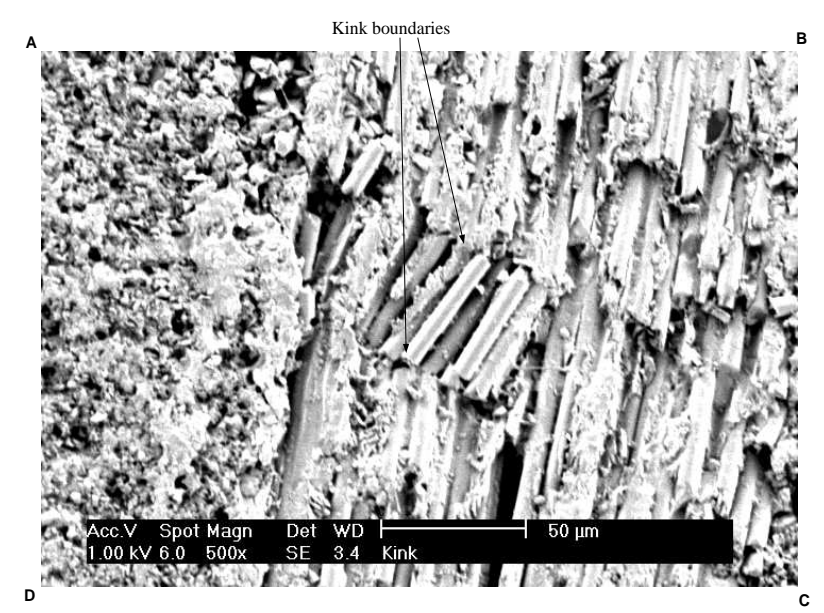

(b) Station 2

Figure 16. Fiber kinking followed by fiber breakage at an axial layer near the top surface. Subsequent interply cracking can also be observed. Notice that the kink band width is about $50 \mu \mathrm{m}$. 


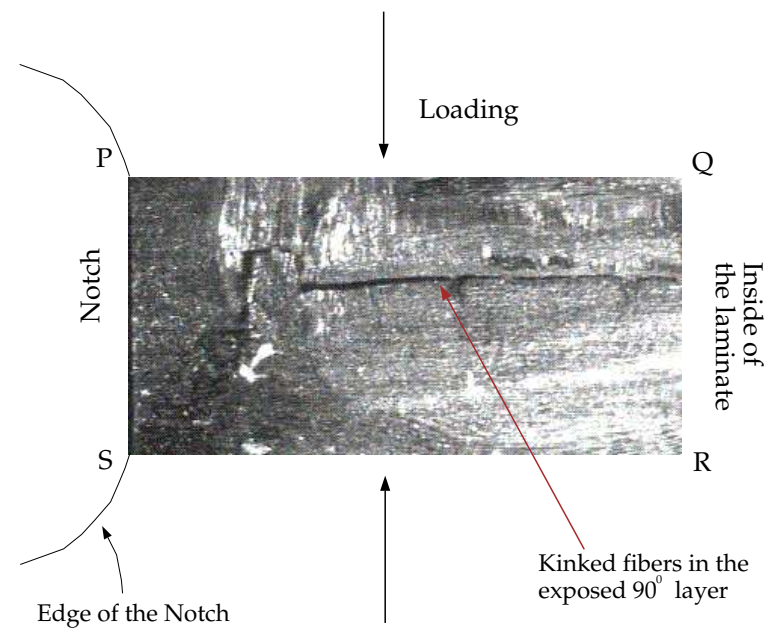

Figure 17. Top view of the kink site PQRS shown in Figure 2. See Figure 16 for the side view - from inside of the laminate. 


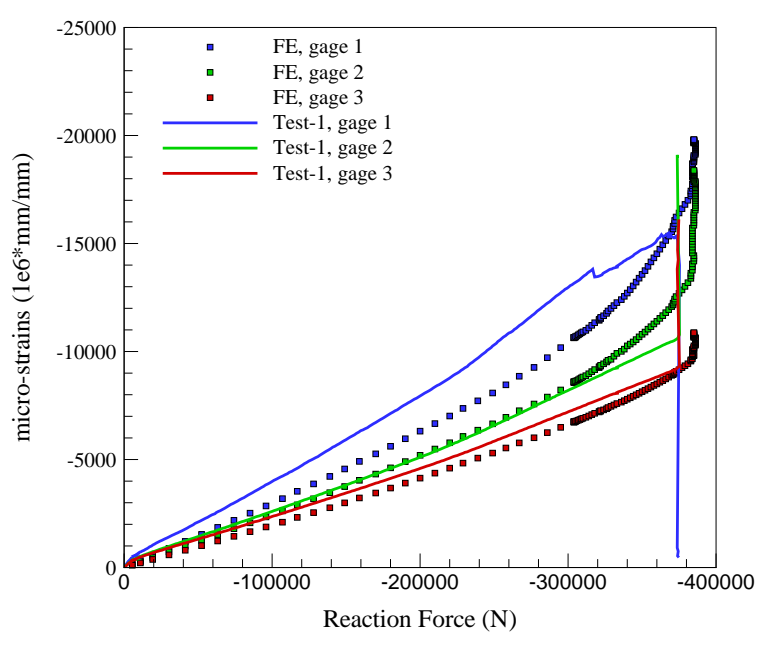

(a) Test-1, left gages

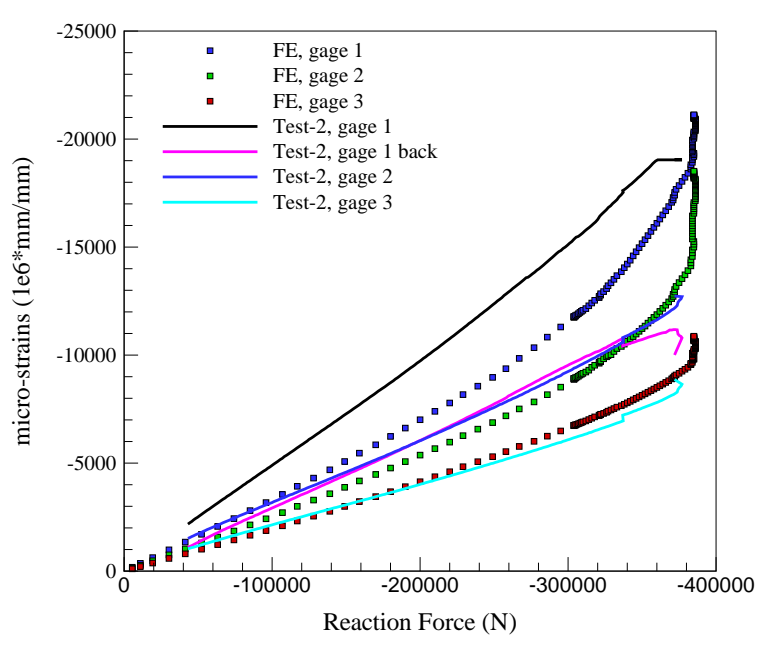

(c) Test-2, left gages

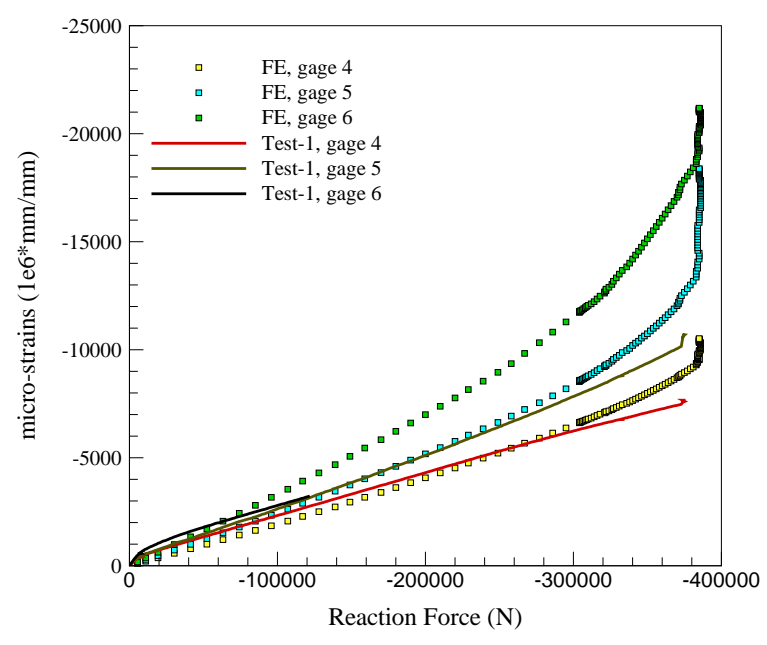

(b) Test-1, right gages

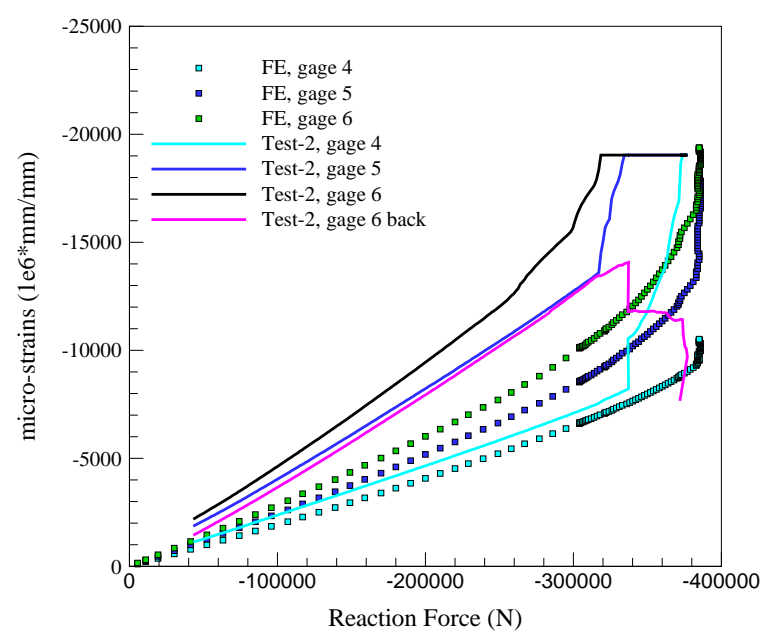

(d) Test-2, right gages

Figure 18. Comparison of measured strain data from experiments with the FE results. Locations of the strain measurement stations on the experimental specimen are given in Table 6. 


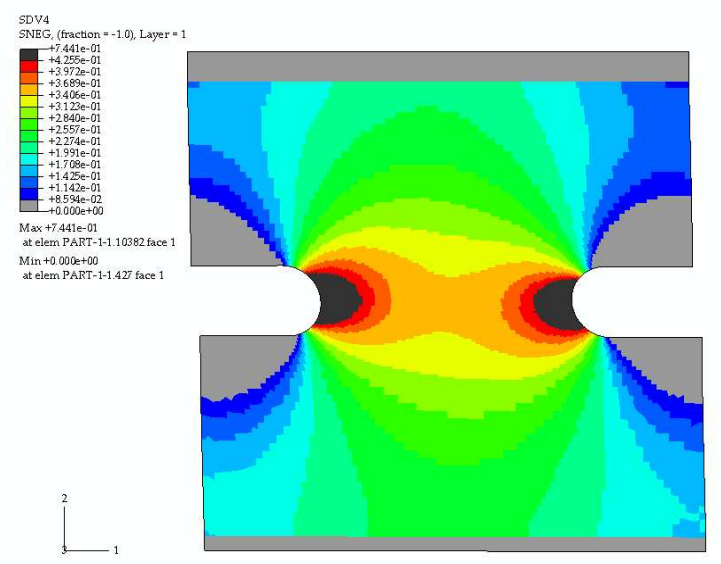

(a) $45^{0}$ Layer

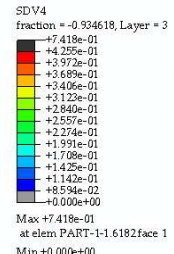

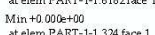
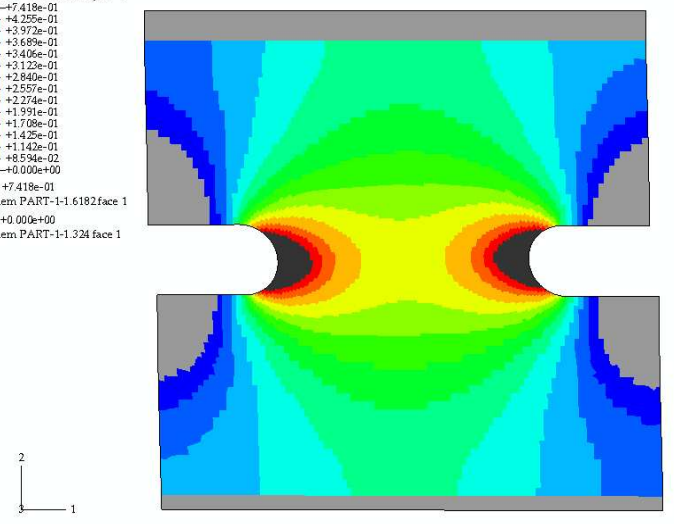

(c) $0^{0}$ Layer

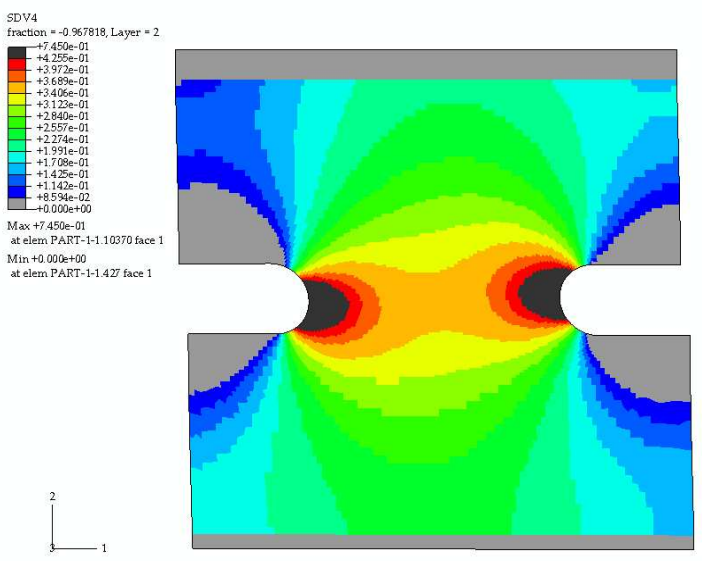

(b) $-45^{0}$ Layer

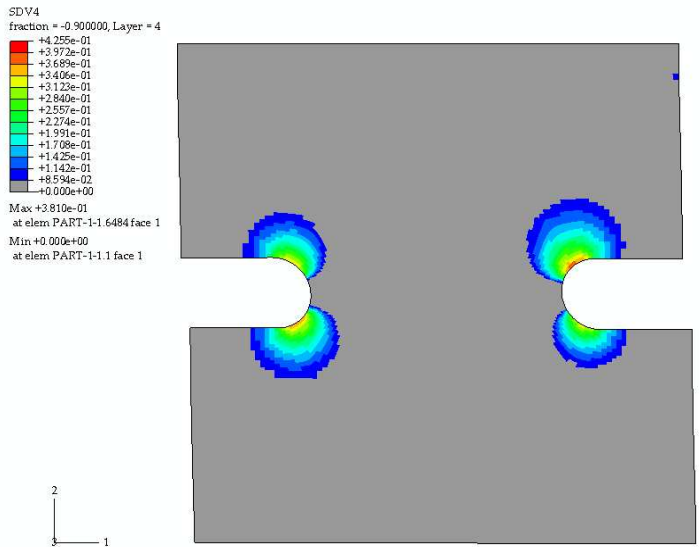

(d) $90^{\circ}$ Layer

Figure 19. Contours of the damage variable $S_{r}$ shown at a global load of $350 \mathrm{KN}$. The contours are shown for the top four layers. Orientation of the layers are given with respect to the global $x$-direction. The dark areas in these contours correspond to $55 \%$ or higher degradation in shear stiffness. 


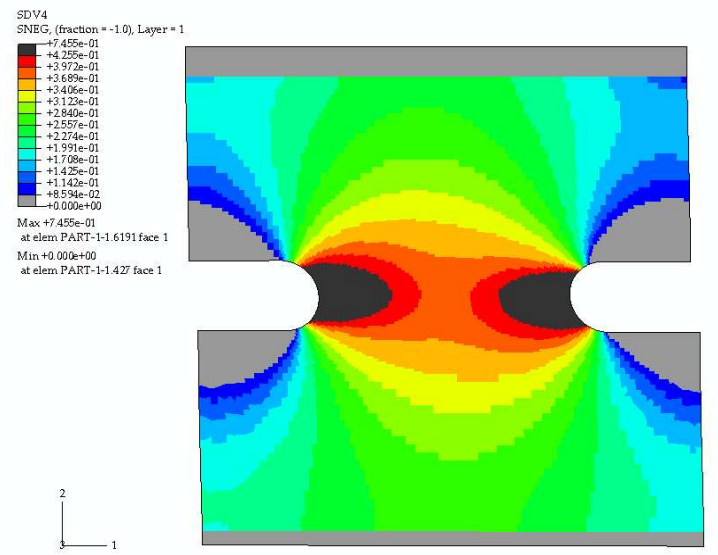

(a) $45^{0}$ Layer

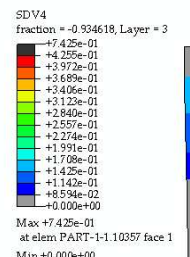

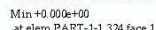

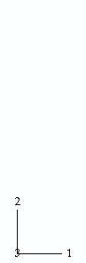

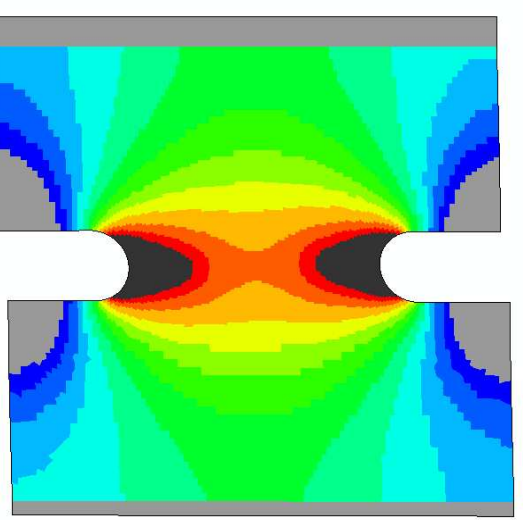

(c) $0^{0}$ Layer

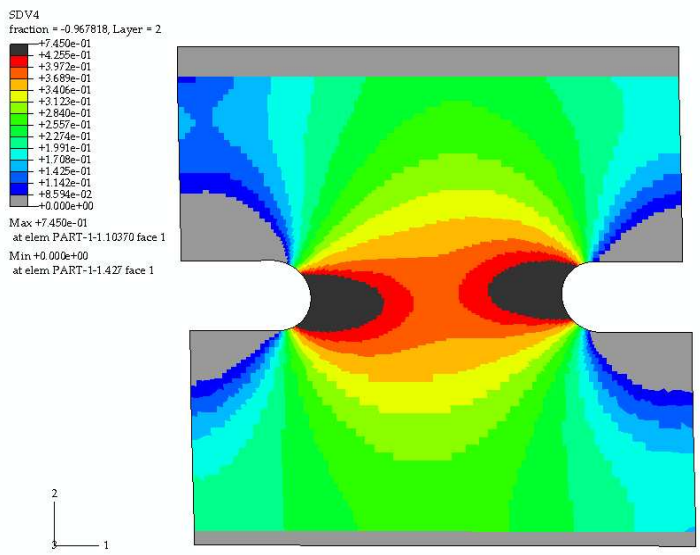

(b) $-45^{0}$ Layer

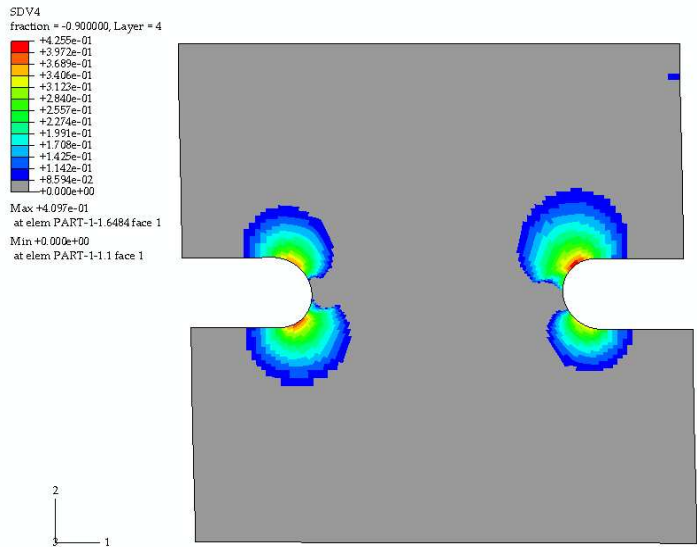

(d) $90^{\circ}$ Layer

Figure 20. Contours of damage variable $S_{r}$ at the peak load of $386 \mathrm{KN}$. The in situ shear modulus of the band of material between the two notch tips is $50 \%$ of their undamaged value. 


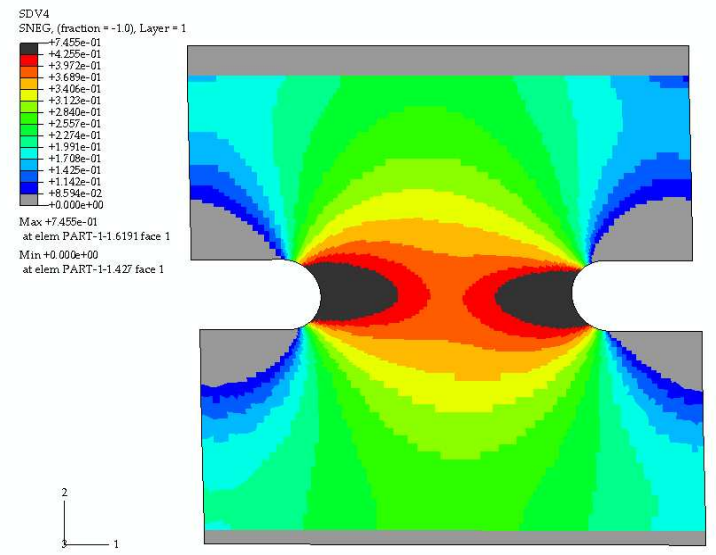

(a) $45^{0}$ Layer

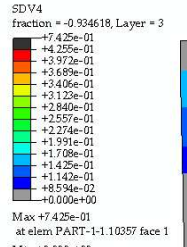

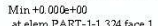

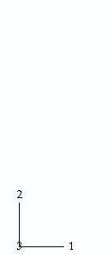

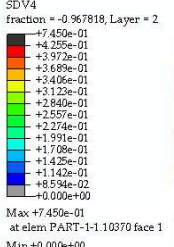

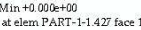

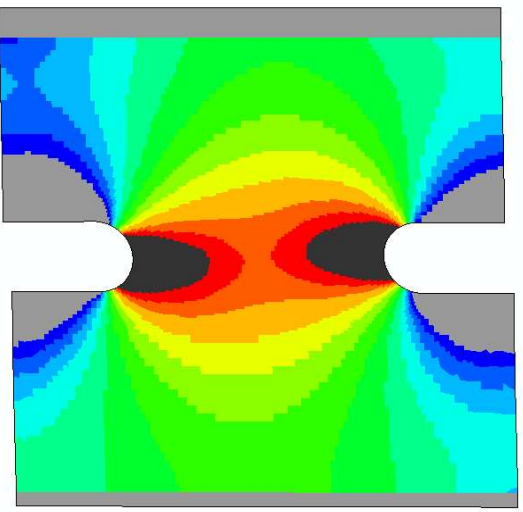

(b) $-45^{0}$ Layer
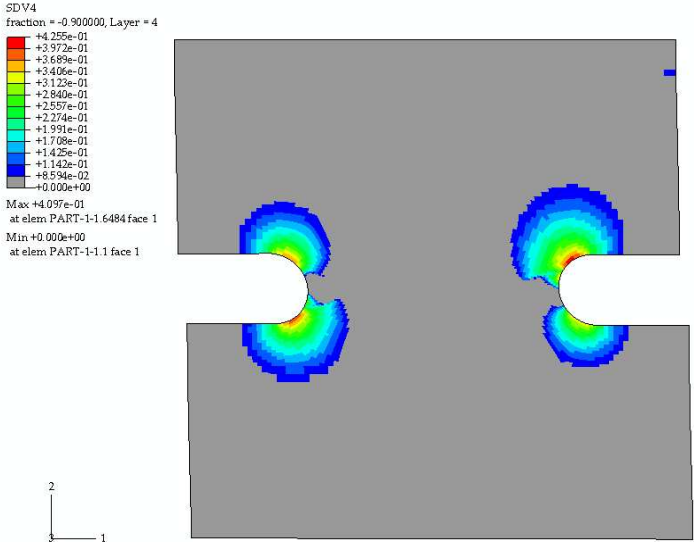

(d) $90^{0}$ Layer

(c) $0^{0}$ Layer

Figure 21. Contours of damage variable $S_{r}$ at a global load of $384 \mathrm{KN}$ (the final point of the FE P- $\Delta$ response curve). 


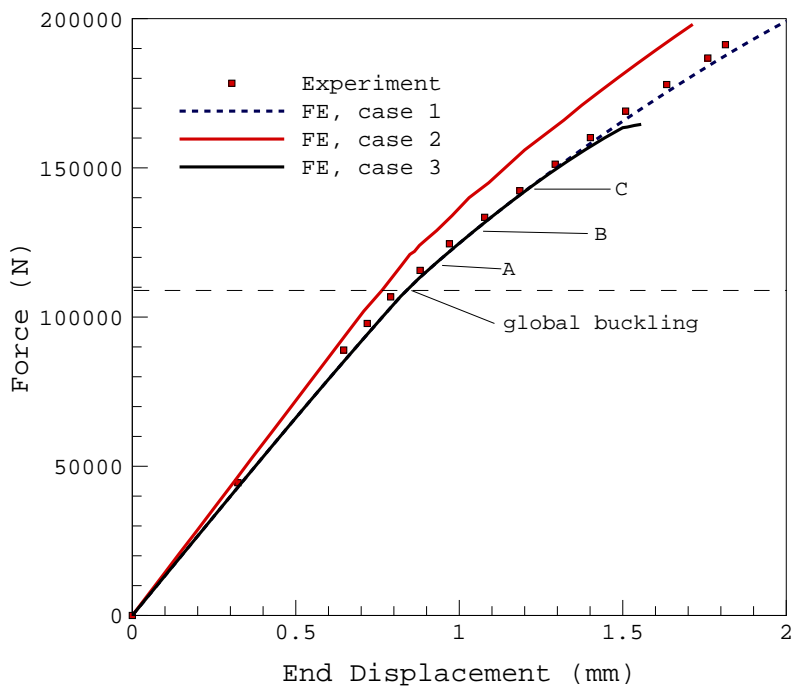

Figure 22. Load-vs.-load point displacement data for the FSP specimen. Locations A, B and C represent various damage events occurring in the laminate and are explained in Table 7.

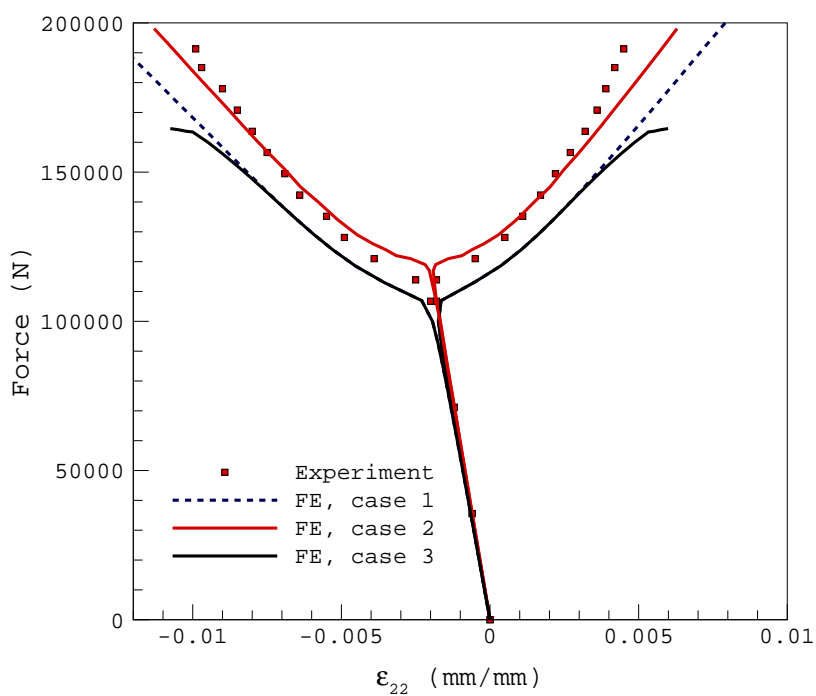

Figure 23. Transverse Strain $\left(\epsilon_{22}\right)$ data at location X (Figure 8) plotted against global load for the FSP specimen.

31 of 38 


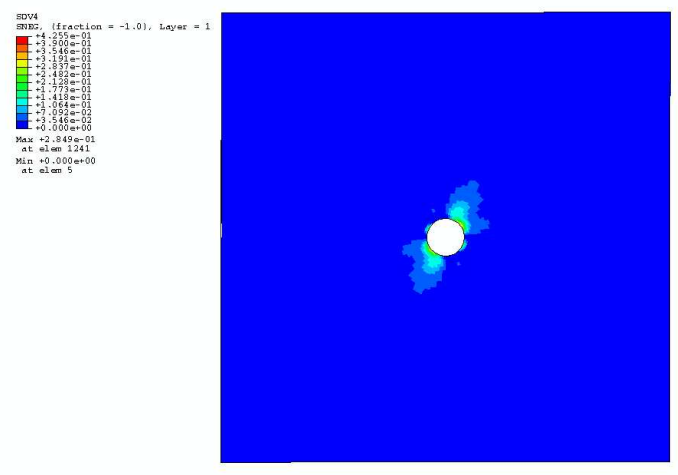

(a) Top $45^{0}$ Layer

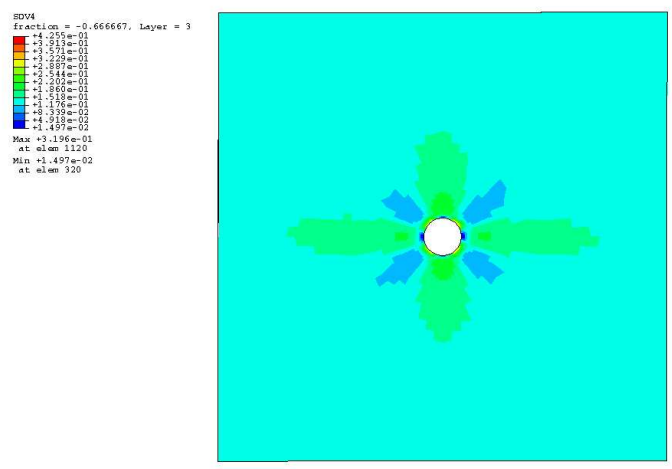

(c) $0^{0}$ Layer

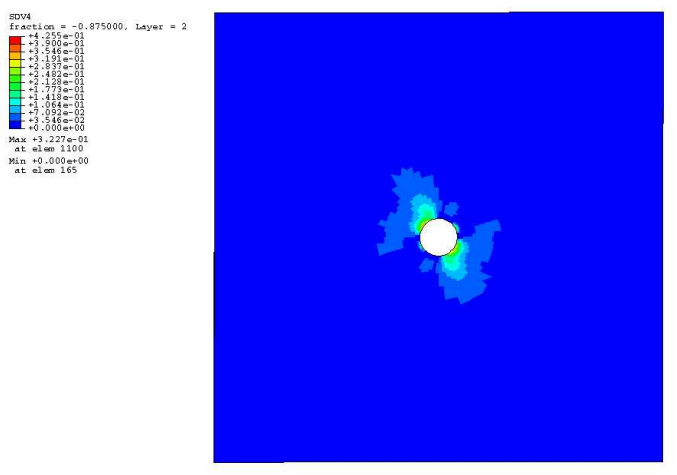

(b) $-45^{0}$ Layer

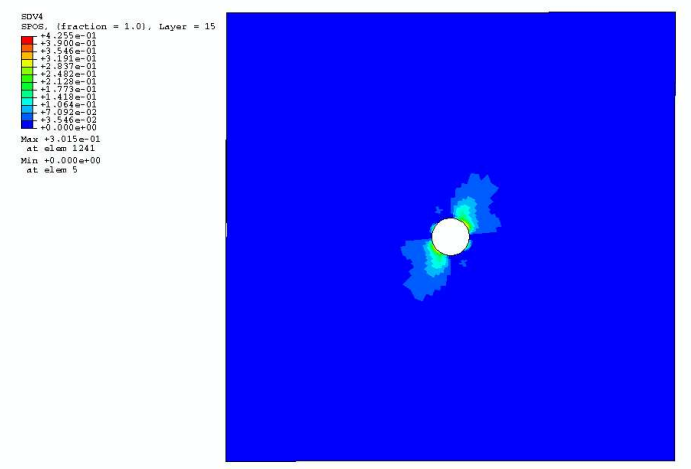

(d) Bottom $45^{0}$ Layer

Figure 24. Contours of the damage variable $S_{r}$ shown at a global load of $100 \mathrm{KN}$. The contours are shown for the top three layers and the bottom layer. Orientation of the layers are given with respect to the global x-direction. The grey areas in these contours correspond to a shear stiffness loss of $55 \%$ or higher. 


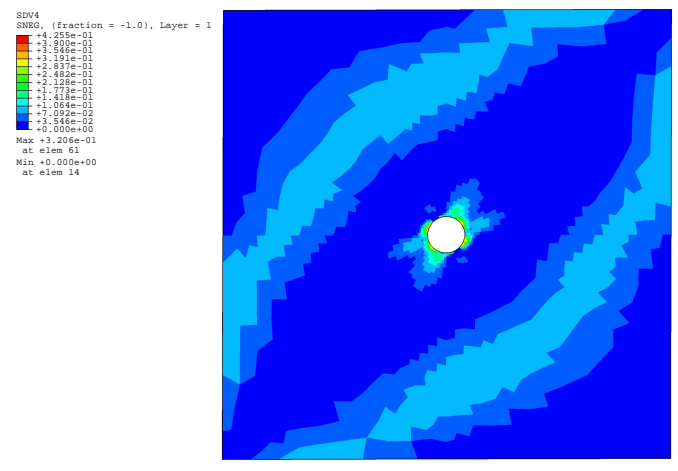

(a) Top $45^{0}$ Layer

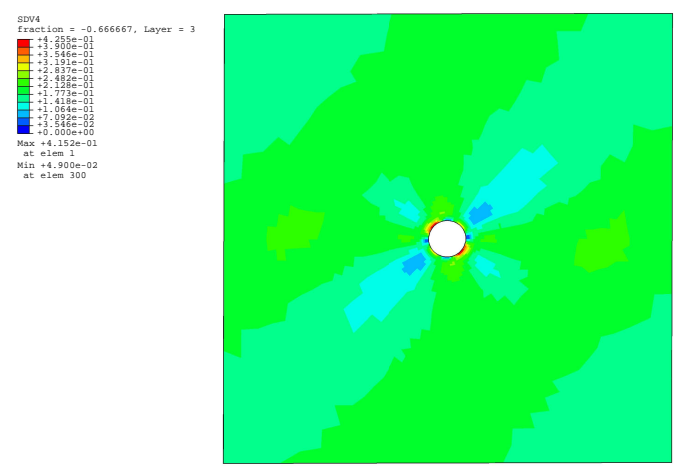

(c) $0^{0}$ Layer

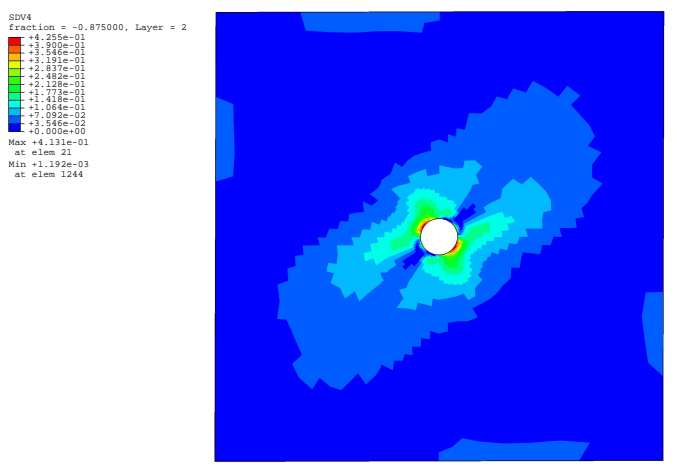

(b) $-45^{0}$ Layer

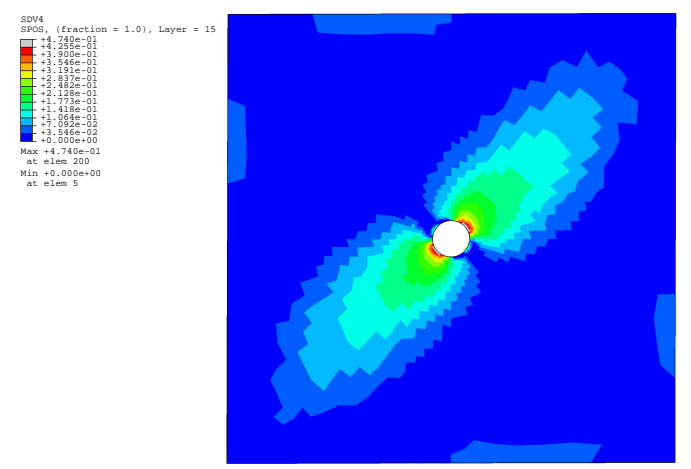

(d) Bottom $45^{0}$ Layer

Figure 25. Contours of damage variable $S_{r}$ at event $A(P=118 \mathrm{KN})$. 


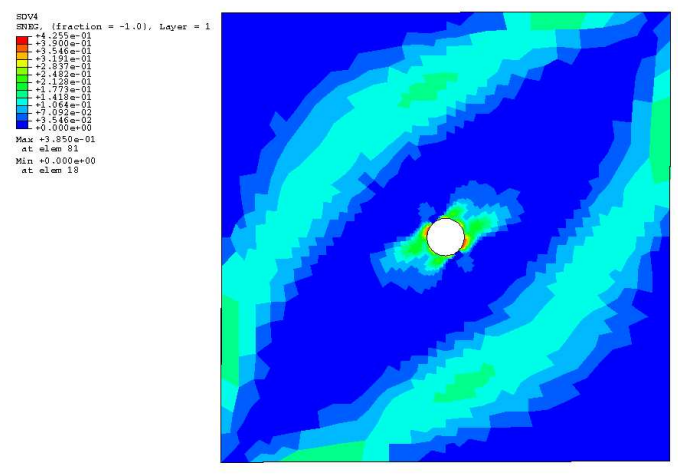

(a) Top $45^{0}$ Layer

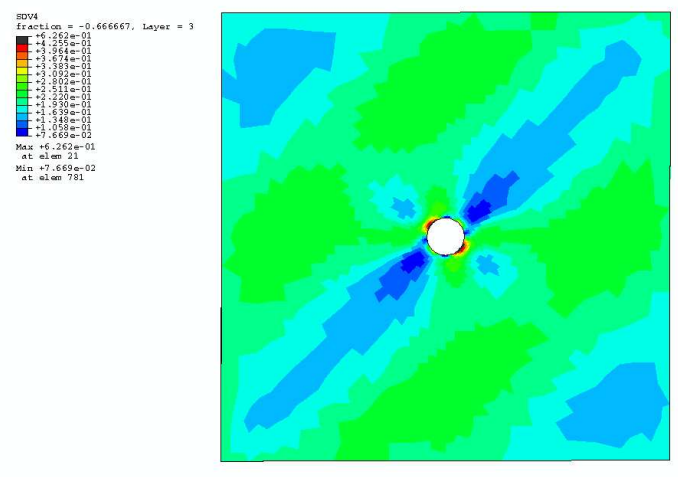

(c) $0^{0}$ Layer

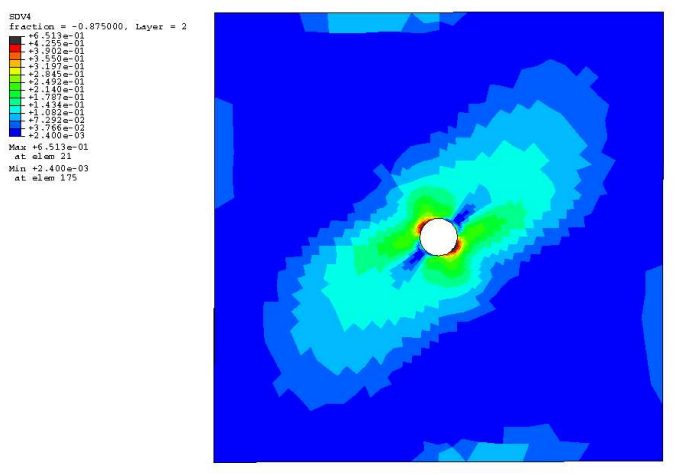

(b) $-45^{0}$ Layer

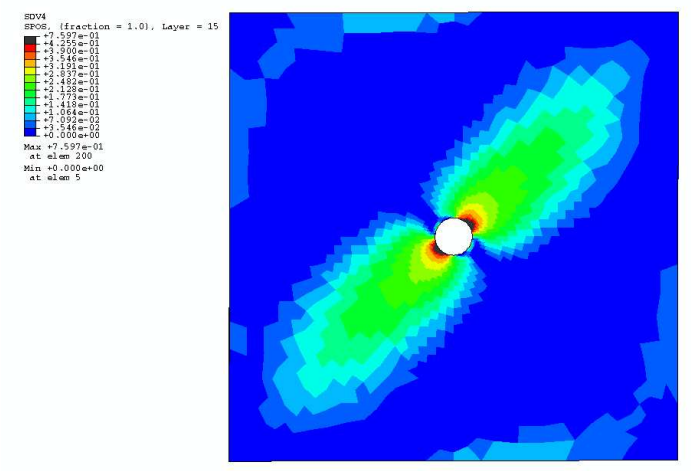

(d) Bottom $45^{0}$ Layer

Figure 26. Contours of damage variable $S_{r}$ at event $\mathrm{B}(\mathrm{P}=129 \mathrm{KN})$. Tensile failure initiates in the topmost layer. 


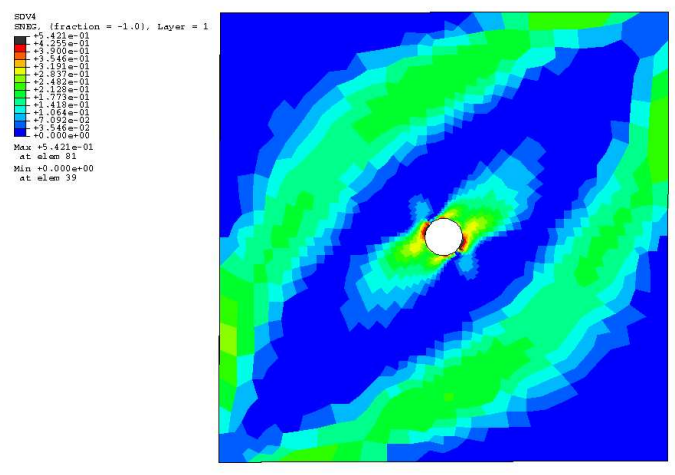

(a) Top $45^{0}$ Layer

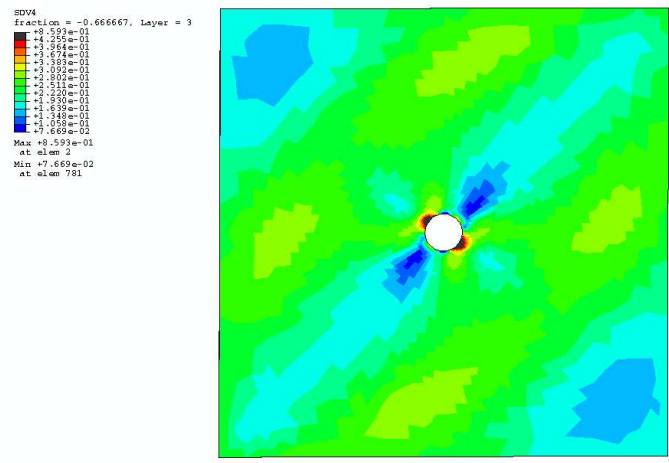

(c) $0^{0}$ Layer

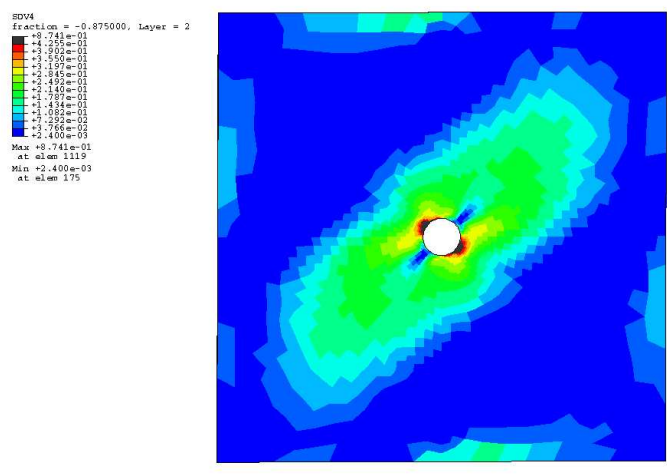

(b) $-45^{0}$ Layer

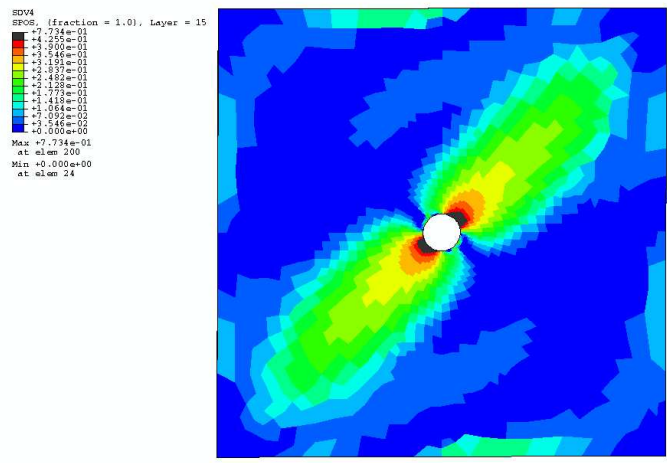

(d) Bottom $45^{0}$ Layer

Figure 27. Contours of damage variable $S_{r}$ at event $\mathrm{C}(\mathrm{P}=143 \mathrm{KN})$. Tensile failure initiates in the bottommost layer. 


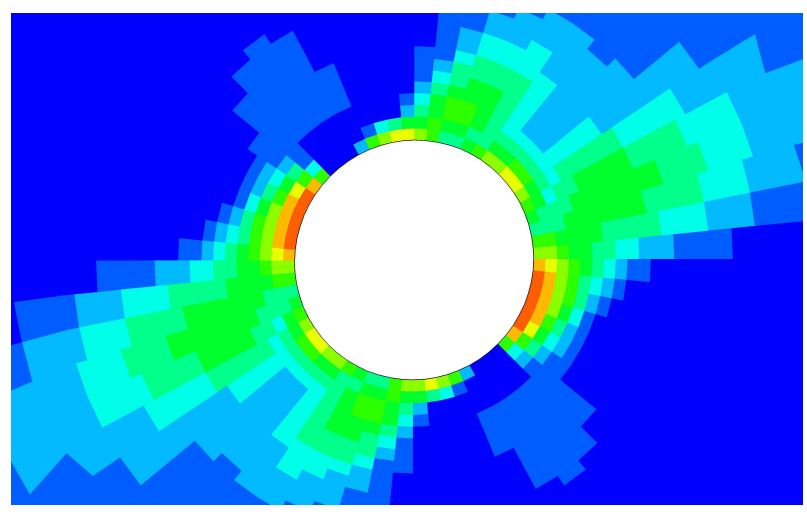

(a) Top $45^{0}$ Layer

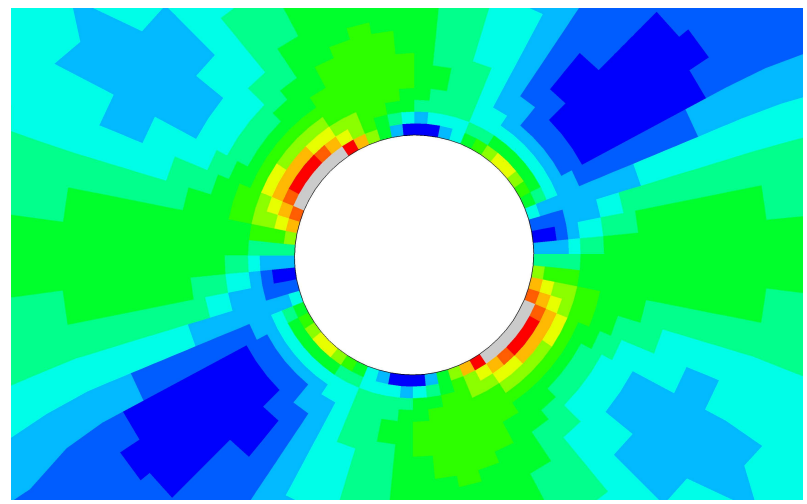

(c) $0^{0}$ Layer

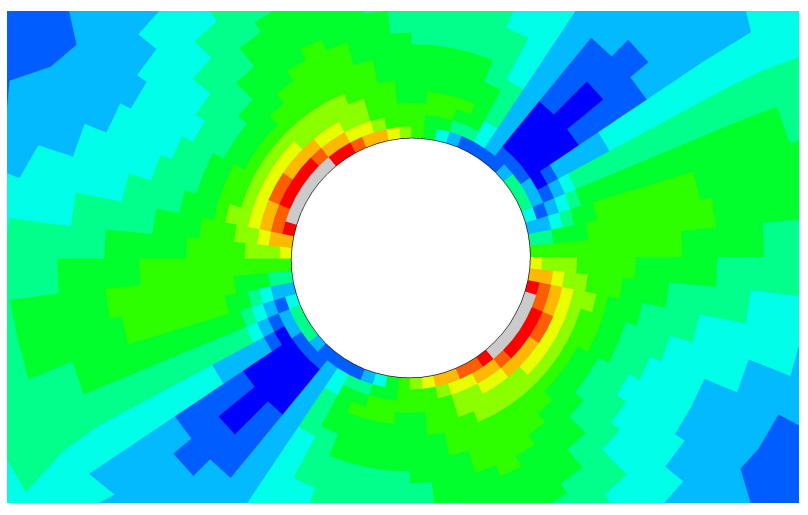

(b) $-45^{0}$ Layer

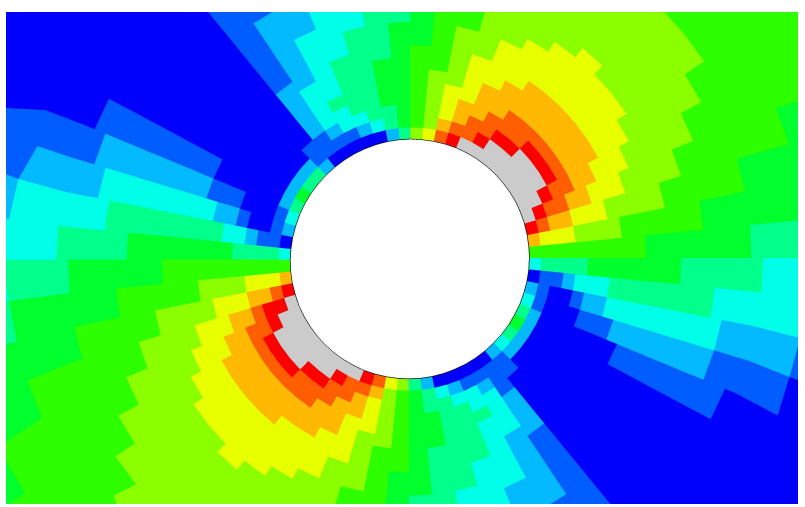

(d) Bottom $45^{0}$ Layer

Figure 28. Close ups of contours of the damage variable $S_{r}$ at event B. The contours show that the inner layers are at a higher level of damage compared to the top layers at the location of tensile failure. The bottom most layer is overall at a higher level of damage compared to the top layer 


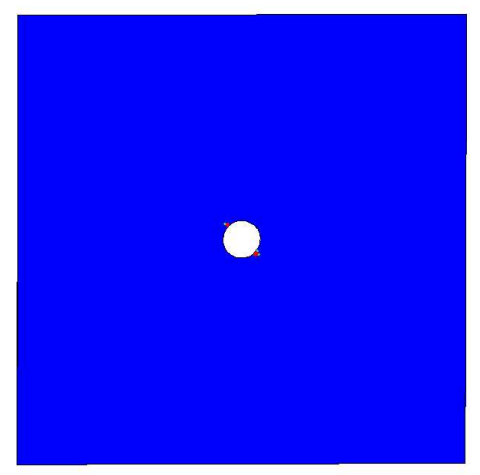

(a) $\mathrm{P}=143 \mathrm{KN}$

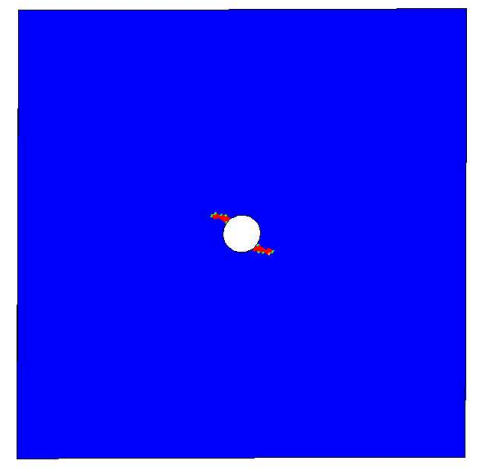

(c) $\mathrm{P}=163 \mathrm{KN}$

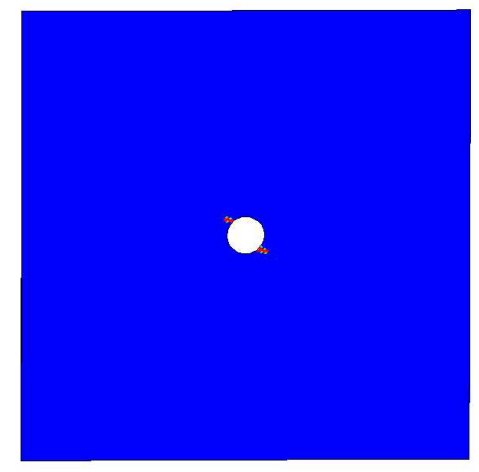

(b) $\mathrm{P}=156 \mathrm{KN}$

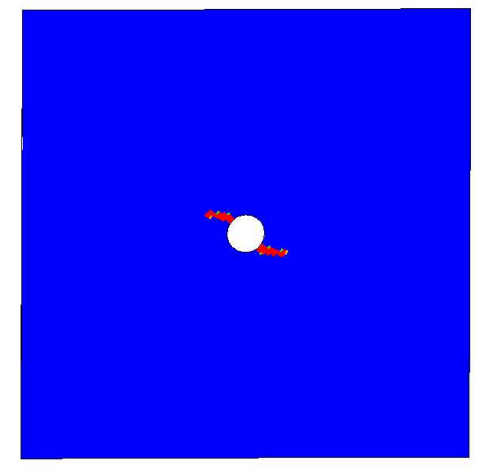

(d) $\mathrm{P}=164 \mathrm{KN}$

Figure 29. Contours of tensile failure in the topmost layer at various points during loading. Tensile failure coupled with off-axis layer shear damage will lead to ultimate failure at the locations indicated. 


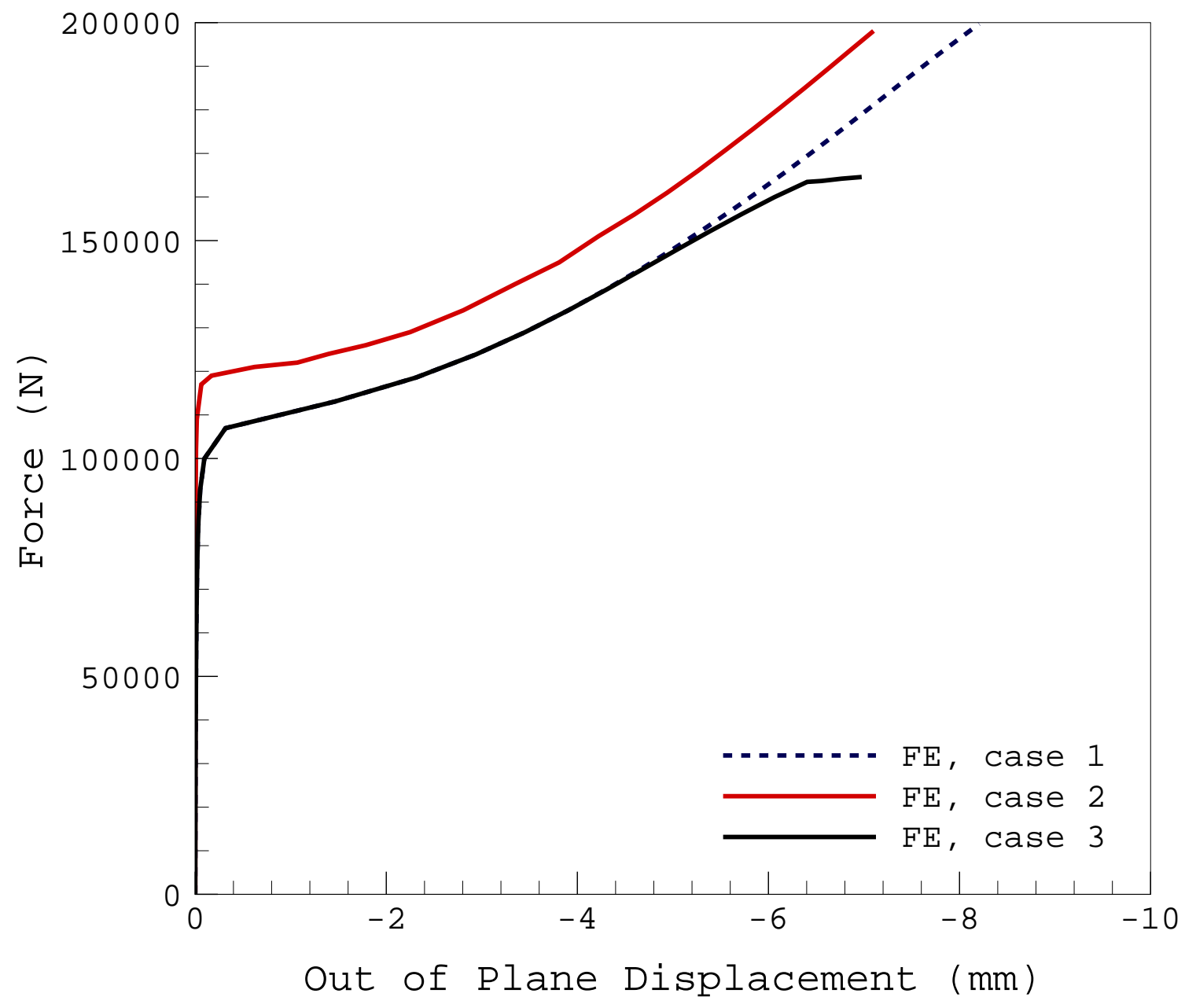

Figure 30. Out-of-plane displacement(w) at the cut-out boundary (Figure 8). 